\title{
Degronomics: Mapping the Interacting Peptidome of a Ubiquitin Ligase Using an Integrative Mass Spectrometry Strategy SUPPORTING INFORMATION
}

\author{
Daniele Canzani, ${ }^{1}$ Domniţa-Valeria Rusnac, ${ }^{2}$ Ning Zheng, ${ }^{2}$ Matthew F. Bush ${ }^{1 *}$ \\ 1 Department of Chemistry, University of Washington, Seattle, WA 98195, USA \\ 2 Howard Hughes Medical Institute, Department of Pharmacology, University of Washington, \\ Seattle, WA 98195, USA. \\ *Address correspondence to: mattbush@uw.edu
}

\section{TABLE OF CONTENTS}

Determining the Binding Affinity of ASDEGEVIVFGG .................................................. S2

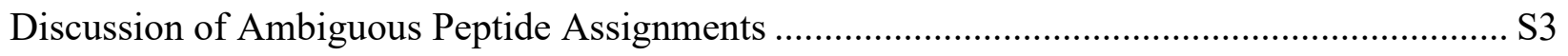

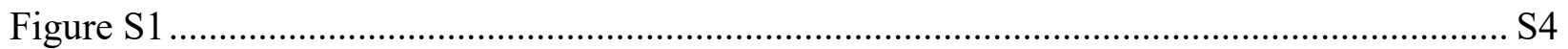

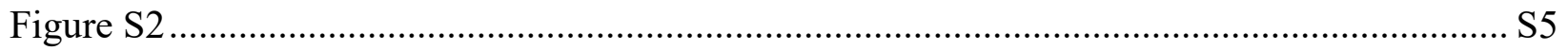

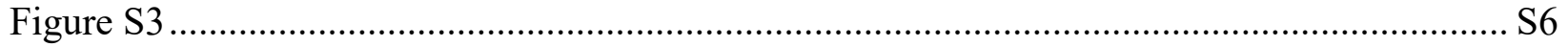

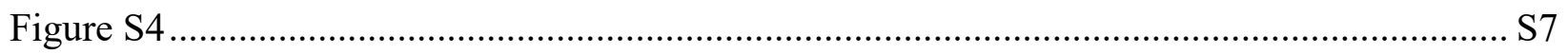

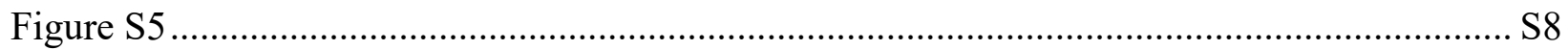

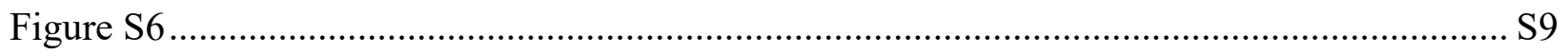

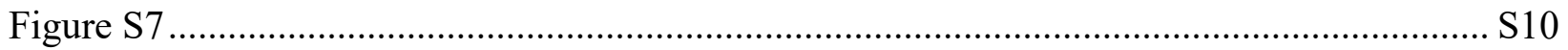

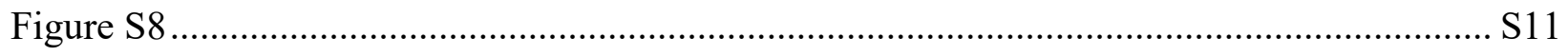

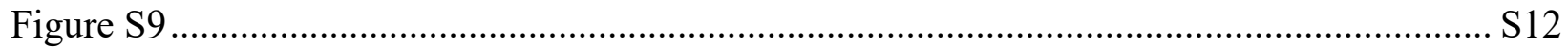

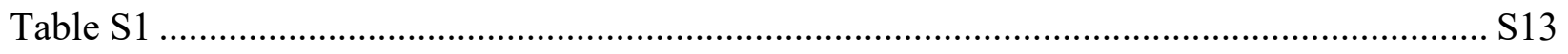

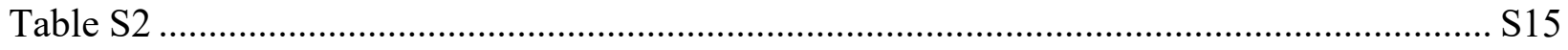

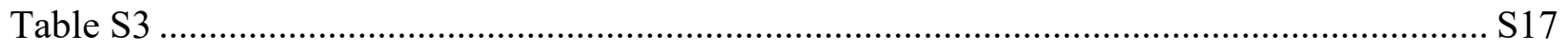

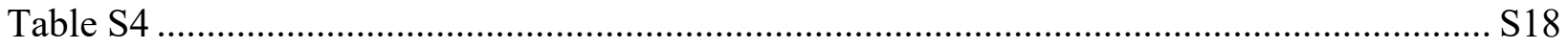

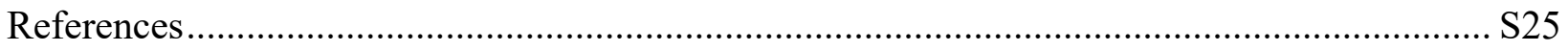

Appendix: Determining Relative Abundances ............................................................ S26 


\section{Determining the binding affinity of ASDEGEVIVFGG}

Binding affinity for the ASDEGEVIVFGG peptide was determined using an AlphaScreen luminescence-based competition assay that was performed using an EnSpire reader (PerkinElmer, Waltham, Massachusetts). GST-tagged KLHDC2 was attached to anti-GST AlphaScreen acceptor beads. Synthetic biotinylated HLRSSPPPMAGG peptide (Bio-Synthesis, Inc., Lewisville, Texas) was immobilized to streptavidin-coated AlphaScreen donor beads. Free ASDEGEVIVFGG peptide was titrated in at various concentrations, which directly competed against the immobilized peptide for binding to KLHDC2. Additional details can be found in Figure S8.

For AlphaScreen competition assays, the kelch repeat domain of human KLHDC2 (amino acids 1-362) was subcloned into the pFastBac vector with an N-terminally fused glutathione-S-transferase (GST) and a TEV-cleavage site. A recombinant baculovirus was produced and amplified three times in Sf9 monolayer cells to produce $\mathrm{P} 4$. The $\mathrm{P} 4$ virus was used to infect Hi5 suspension insect cell cultures to produce the recombinant GST-KLHDC2 protein. The cells were harvested 2-3 days after infection, re-suspended and lysed in lysis buffer ( $20 \mathrm{mM}$ Tris, $\mathrm{pH} 8.0,200 \mathrm{mM} \mathrm{NaCl}, 5 \mathrm{mM}$ dithiothreitol) in the presence of protease inhibitors using a microfluidizer. The GST-KLHDC2 protein was isolated from the soluble cell lysate by Pierce ${ }^{\mathrm{TM}}$ Glutathione Agarose (Thermo Fisher Scientific). The GST tagged KLHDC2 was further purified by Q Sepharose High Performance resin (GE Healthcare). The $\mathrm{NaCl}$ eluates were subjected to Superdex-200 size-exclusion chromatography. 


\section{Discussion of ambiguous peptide assignments.}

The strategy used to identify peptides is described in the Methods. Most reported peptide sequences are based on very high probabilities, in terms of both their Comet probabilities and their PeptideProphet iprobabilities (Table S1). Among the peptides that are assigned to specific proteins, we included some peptides based on low-probability peptide-spectrum matches, but for which there were N-terminal extensions of that sequence identified through high-probability peptide-spectrum matches. In those cases, the fragmentation spectra were inspected individually and deemed to be consistent with the reported sequence (in terms of accurate masses and that the most intense fragments could be assigned to the expected $b$ and $y$ ions). These spectra received low probabilities because of the inclusion of similar sequences in the protein database; those results on their own would not have been adequate for peptide identification. However, the simplest explanation is that those $\mathrm{LC}-\mathrm{MS}^{2}$ results are attributable to a N-terminal truncation of a longer protein fragment.

Other peptide-spectrum matches, particularly for lower mass peptides, were not adequate for assignment to a specific peptide or protein. For example, LPEFGG is present within 5 E.coli proteins, so a definitive protein match is not possible. Because leucine and isoleucine are not differentiated in these experiments, this problem is compounded for short peptides that contain one of those residues. That is, the fragmentation spectra underlying those assignments are ambiguous in terms of both peptide sequence (leucine versus isoleucine) and originating protein. 


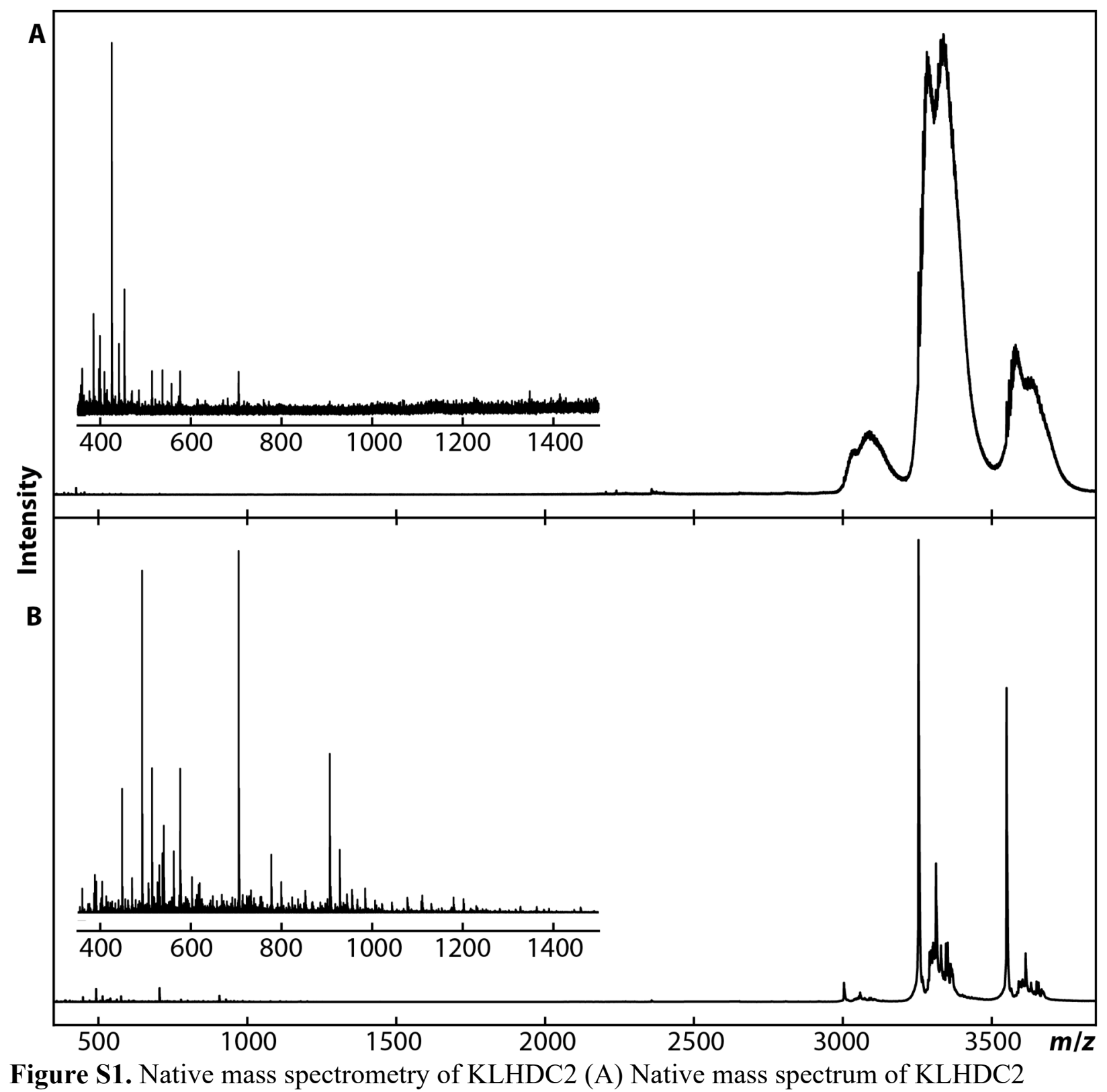

acquired without activation. (B) Native mass spectrum of KLHDC2 using the quadrupole profile that favors ions with $m / z>3000$ followed by activation in the trap, which caused a number of comparatively low- $m / z$ peptide ions to be released from KLHDC2:peptide complexes. The sum of the total ion abundance over $400-1500 \mathrm{~m} / \mathrm{z}$ is 2.4 times larger than that of A, which demonstrates that peptides ions are significantly more abundant in the CID activated spectrum. 


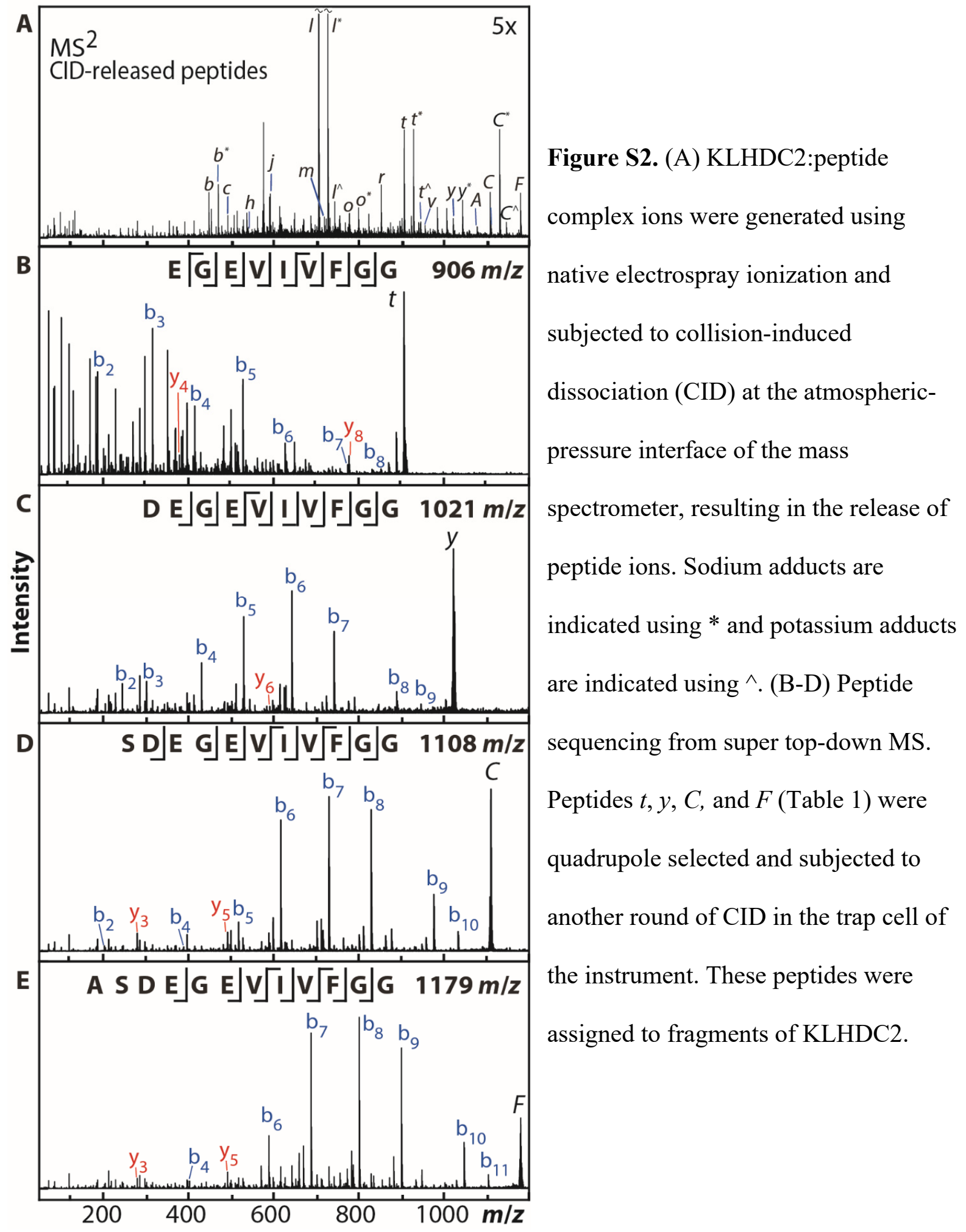




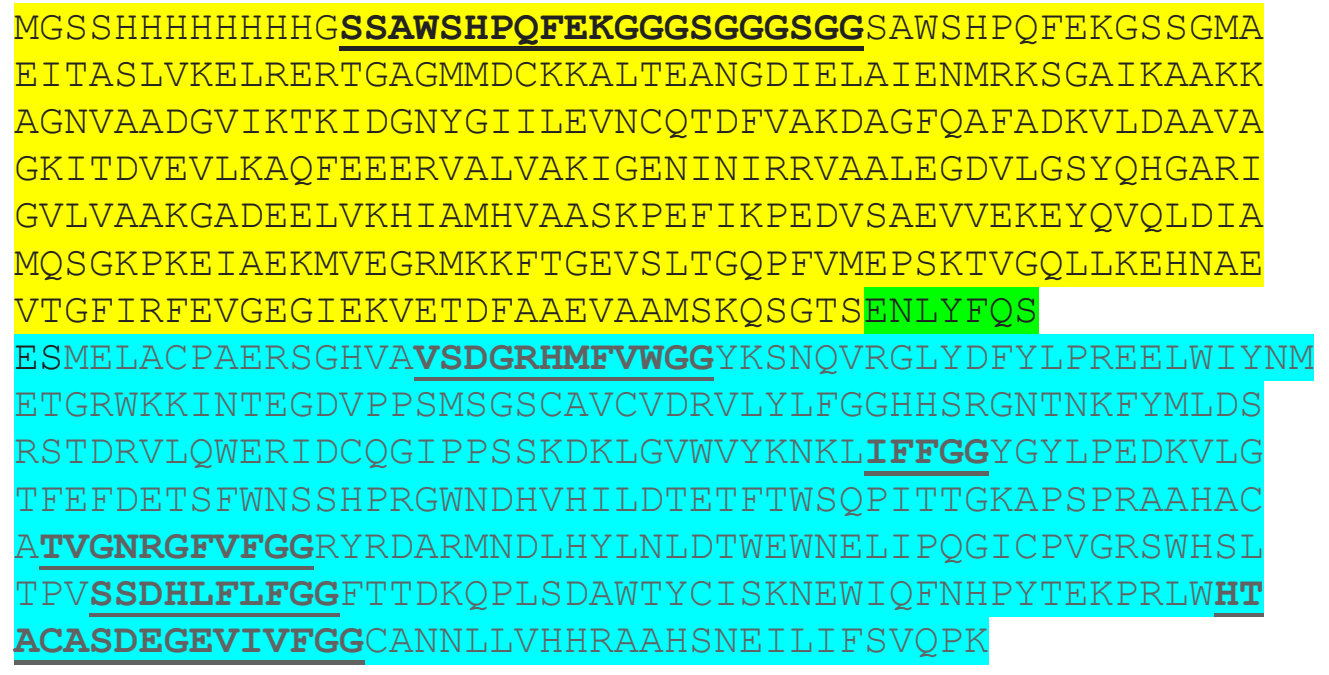

Figure S4. Sequence of His-TSF-KLHDC2. His-TSF solubility tag (yellow) was cleaved from KLHDC2 (cyan) using TEV protease at the cleavage site (green) during purification. Peptides that were found to be bound to KLHDC2 are underlined. 


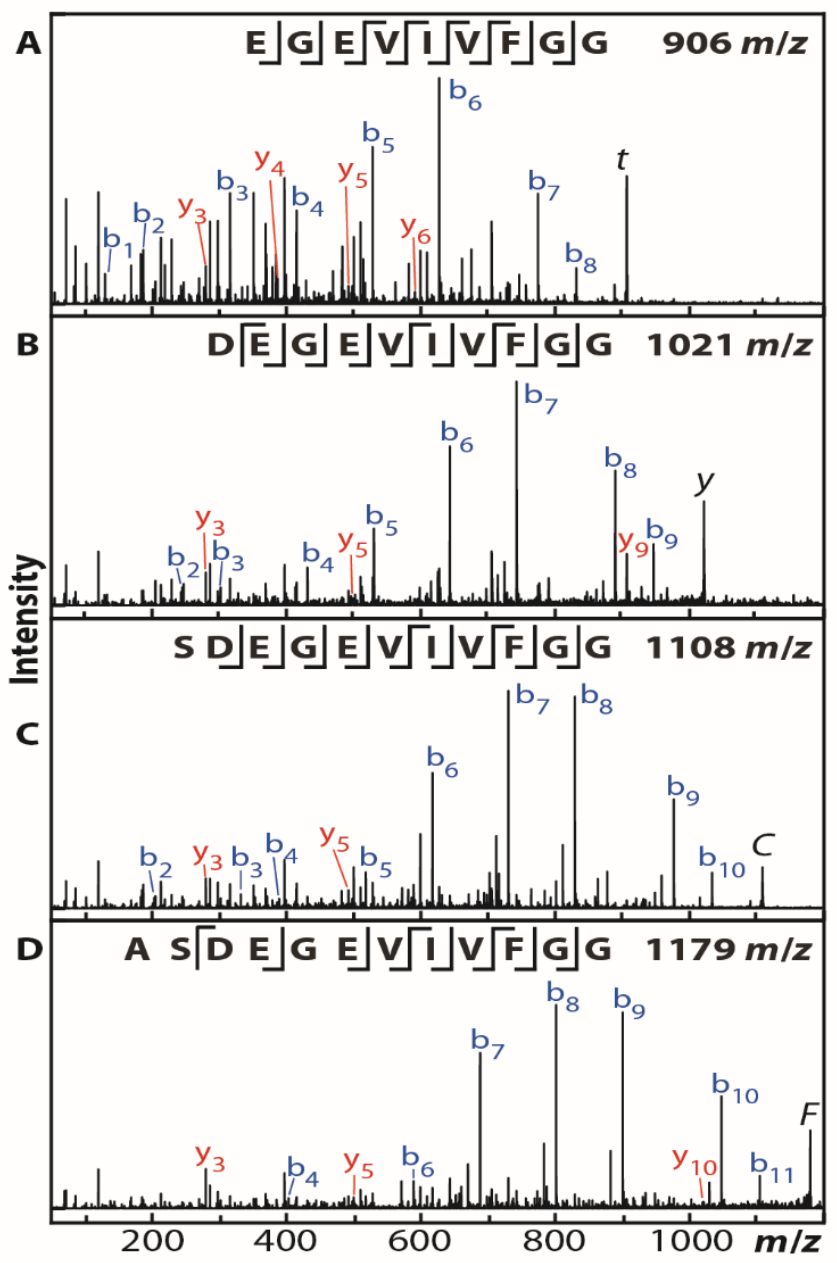

Figure S4. (A-D) Peptide sequencing from heat destabilization. Peptides that were released in solution by placing the sample for native MS into a heat block set at $55^{\circ} \mathrm{C}$ were quadrupoleselected and subjected to CID in the trap cell of the instrument. These four peptides originated from KLHDC2 and have the same sequence, which features the C-terminal diglycine degron that is recognized by KLHDC2. 


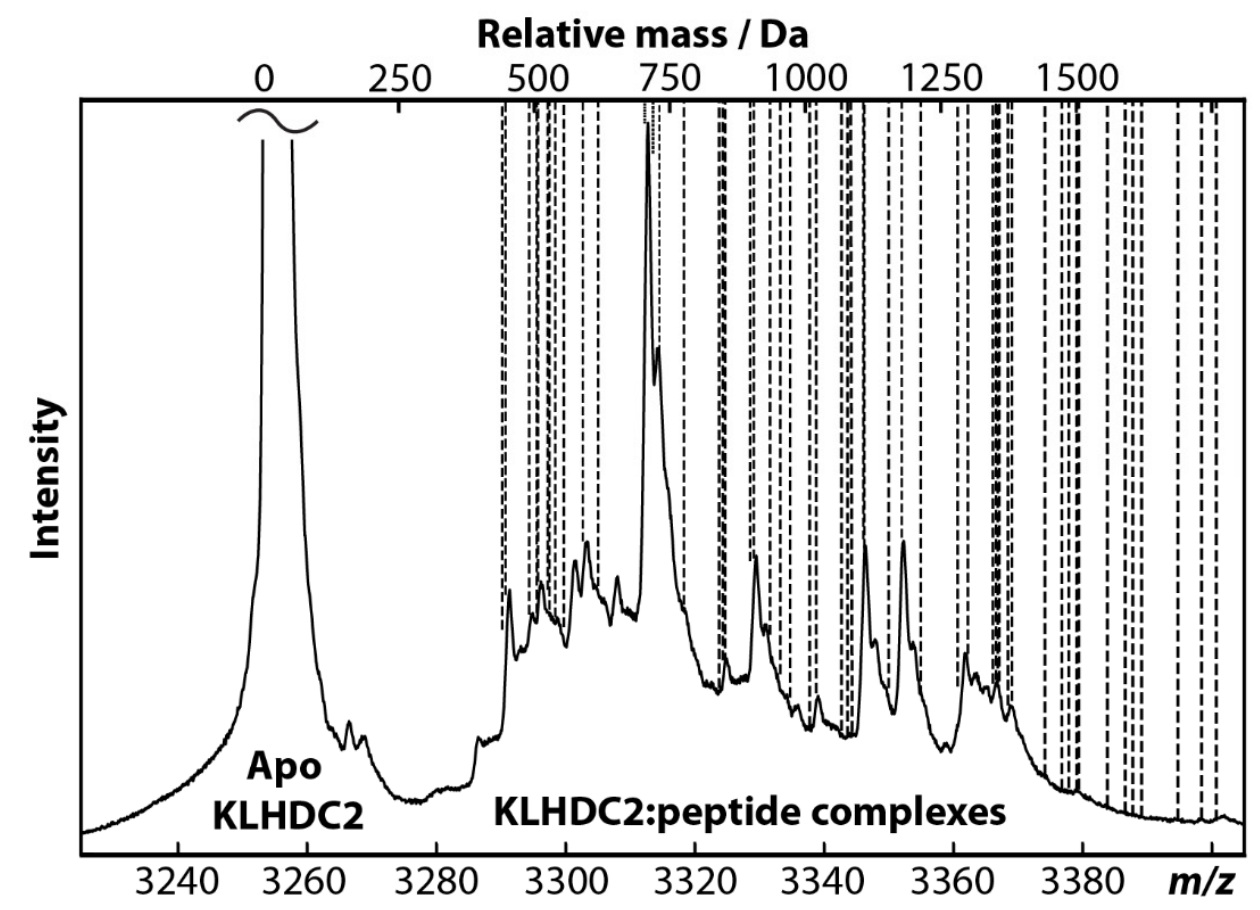

Figure S5. Peptides identified using the integrative mass spectrometry approach are mapped to the native spectrum of the $12+$ ion of KLHDC2. The top axis shows the mass of the peptides, relative to apo KLHDC2. 


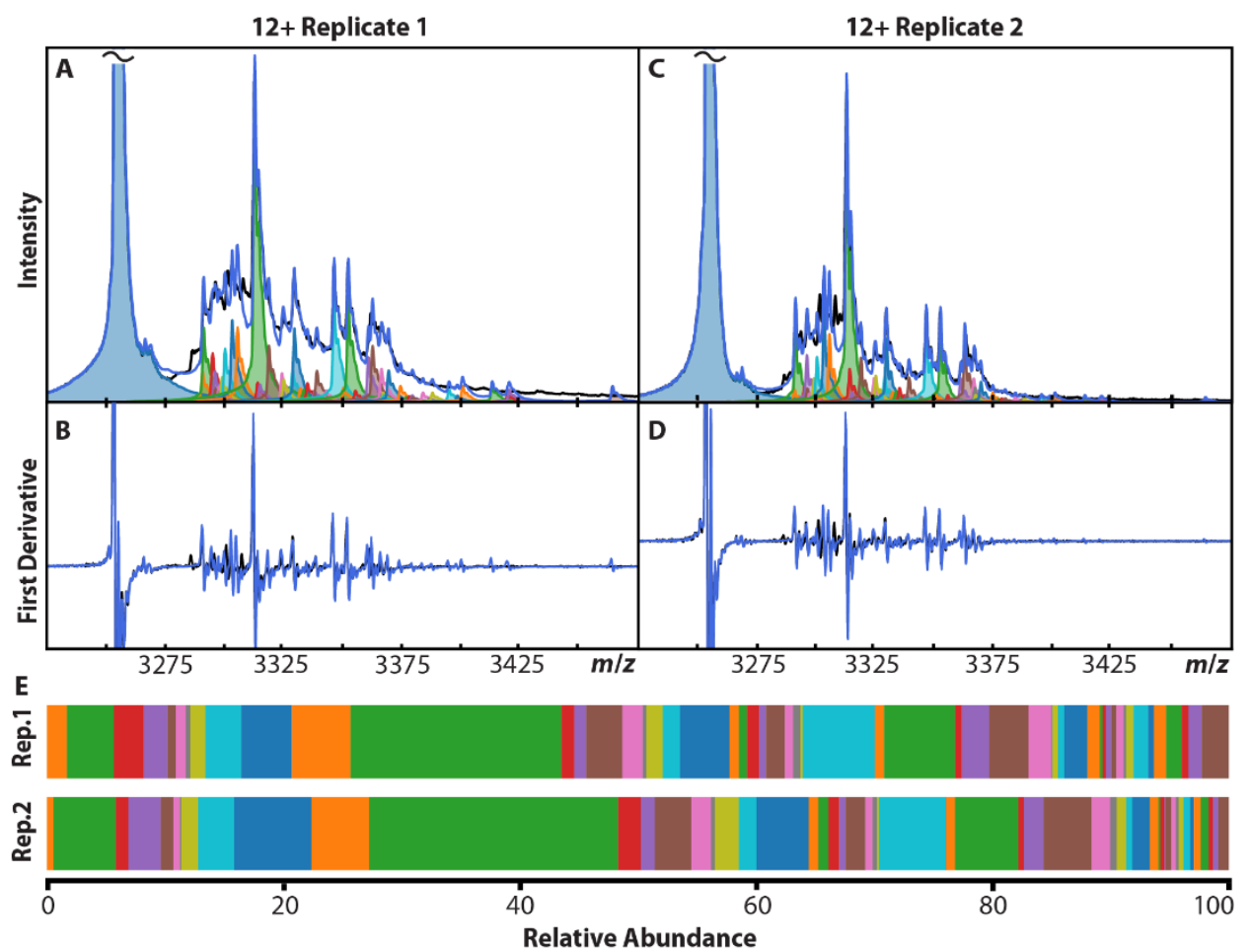

Figure S6. Relative peptide abundance between replicates. (A) The native mass spectrum of $12+$ KLHDC2 (also shown in Fig. 4B), with contributions from each identified peptide estimated using apo KLHDC2 as an internal reference. The sum of the residuals between the experiment (black trace) and the models (colored traces) were minimized to provide optimized relative peptide abundances. (B) The first derivatives of the experimental spectrum (black trace) and the sum of the modelled components (blue trace) are also highly correlated. (C) The native mass spectrum of $12+$ KLHDC2 from an additional sample replicate is shown (from the same purification, but prepared for native MS and analyzed on separate days). (D) The first derivatives of the experimental spectrum (black trace) and the sum of the modelled components (blue trace) from the second replicate. (E) The average relative peptide abundances (from the $11+, 12$ and $13+$ charge states) for each of the technical replicates. 


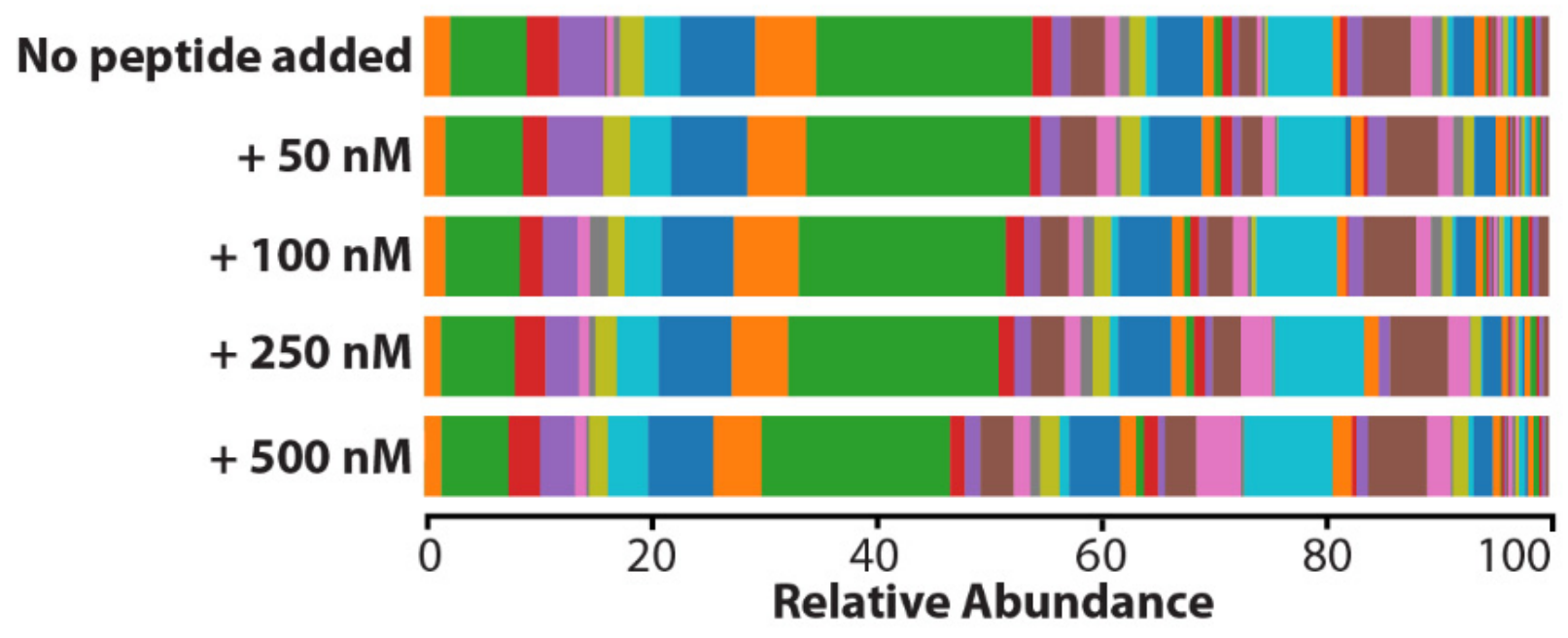

Figure S7. Adapted from the titration data reported in Figure 5. Relative abundances for all peptides other than peptide $F$ (ASDEGEVIVFGG), which was titrated into the sample at the concentrations reported in each row. The abundances of these peptides are relatively consistent between measurements. 


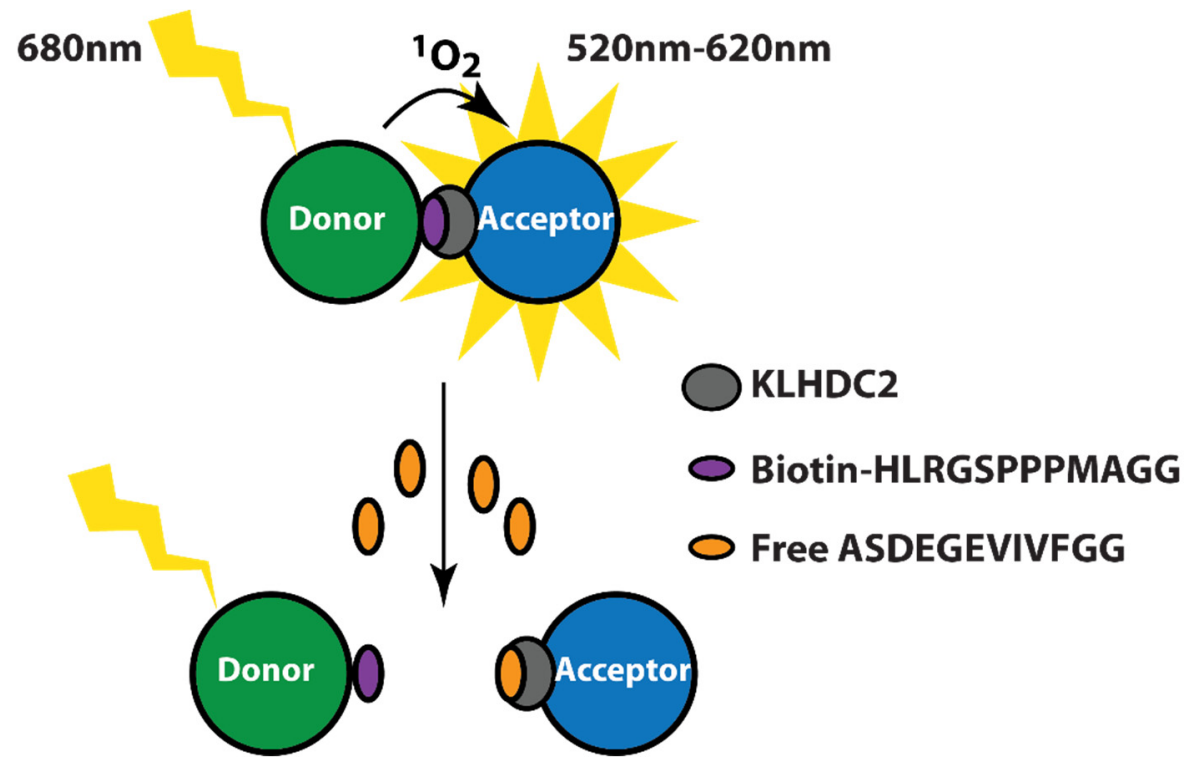

Figure S8. AlphaScreen-based binding assay. Donor and acceptor beads are fused with synthesized biotinylated-HLRGSPPPMAGG peptide from SelK and GST-fused KLHDC2, respectively. When the donor bead is excited by a $680 \mathrm{~nm}$ laser, singlet oxygen is produced from ambient oxygen. Singlet oxygen reacts with the acceptor bead, which results in the emission of photons. The half-life of singlet oxygen in aqueous media is very low, so the beads must be in close proximity (immobilized KLHDC2 and immobilized peptide must be bound together) for the reaction and luminescence to occur. The experiment was conducted with $0.12 \mathrm{nM}$ of GSTKLHDC2 and $1.7 \mathrm{nM}$ biotinylated SelK peptide in the presence of $5 \mu \mathrm{g} \mathrm{mL}{ }^{-1}$ donor and acceptor beads in a buffer of $25 \mathrm{mM}$ HEPES, $\mathrm{pH}$ 7.5, $100 \mathrm{mM} \mathrm{NaCl}, 1 \mathrm{mM}$ DTT, 0.1\% Tween-20, and $0.05 \mathrm{mg} \mathrm{mL}^{-1}$ bovine serum albumin. Free ASDEGEVIVFGG was titrated in at various concentrations $(0.15 \mathrm{nM}$ to $9 \mu \mathrm{M})$, and luminescence was measured. Precise concentration of the stock peptide was determined by amino acid analysis (TAMU Protein Chemistry Lab, Texas A\&M University). The experiment was done in triplicates. $\mathrm{IC}_{50}$ value was determined using nonlinear curve fitting of the dose response curve generated with Prism 4 (GraphPad, San Diego, California). $\mathrm{IC}_{50}$ is approximately equal to $\mathrm{K}_{\mathrm{d}}$ due to the very low concentration of immobilized components in these experiments. 


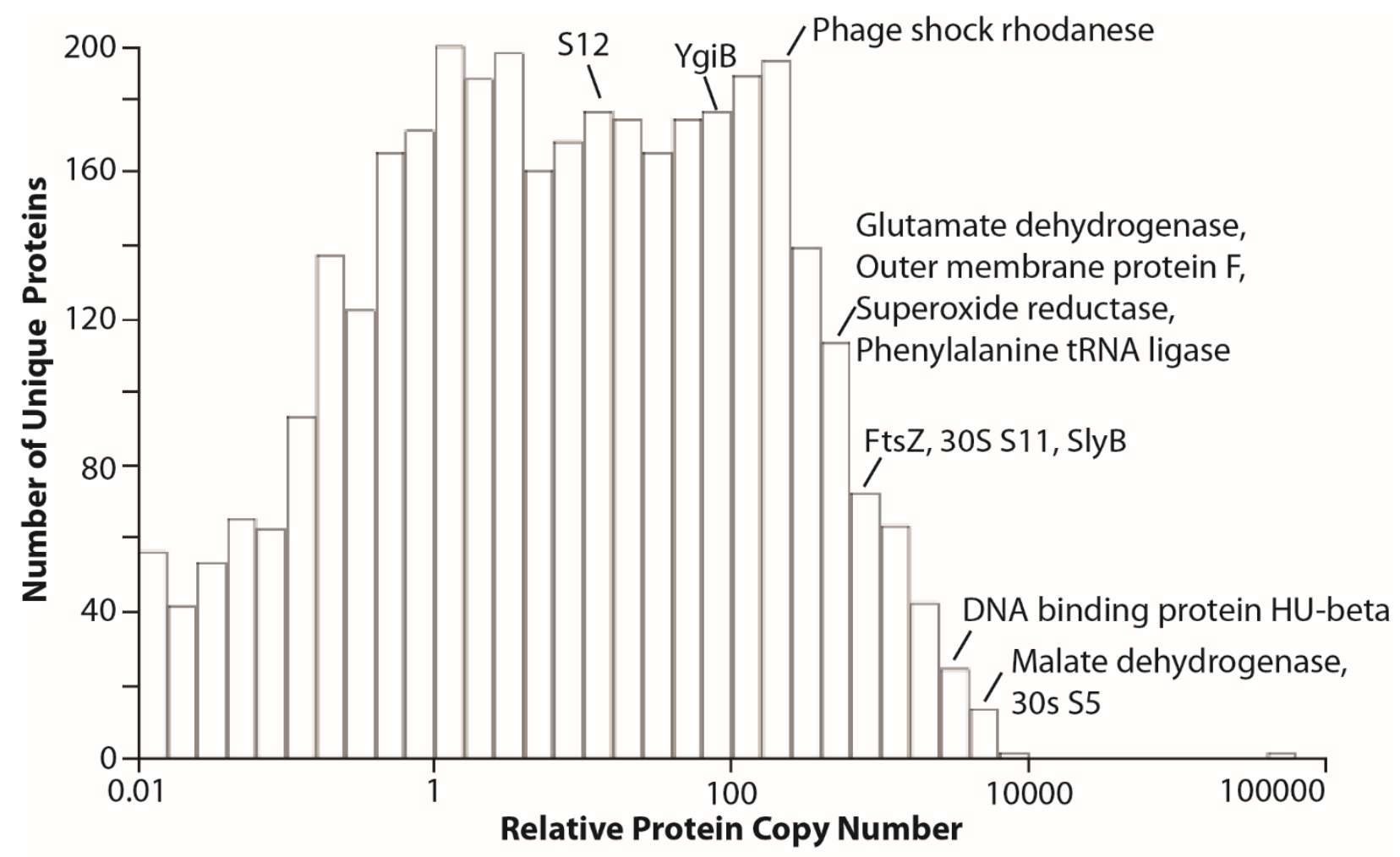

Figure S9. Abundance histogram of all E. coli $\mathrm{K}-12$ proteins based on the meta analysis in PaxDB. ${ }^{1}$ The relative protein copy number is the number of copies of a given protein per one million total proteins in E. coli cells. Peptides from several E. coli proteins were identified, which range in in cellular copy number over several orders of magnitude. This figure is adapted from https://pax-db.org/species/511145 
Table S1. Peptide identifications, PeptideProphet iprobability, Comet probability scores, and relative abundance from XPRESS.

\begin{tabular}{|c|c|c|c|c|c|c|c|c|c|}
\hline Protein & Uniprot ID & Peptide & ID & $m / z$ & $\mathbf{z}$ & iprobability & probability & $\begin{array}{l}\text { XPRESS } \\
\text { Rel. Ab. } \\
\text { (\%) }\end{array}$ & Representative Spectrum \\
\hline \multirow{18}{*}{ KLHDC2 } & \multirow{18}{*}{ Q9Y2U9 } & VSDGRHMFVWGG & $J$ & 449.8809 & 3 & 0.999994 & 0.9999 & 0.0167 & 180912_KLHDC2_Ecoli_01.11299.11299.3 \\
\hline & & SSDHLFLFGG & $A$ & 540.7626 & 2 & 0.321751 & 0.0315 & 0.0064 & 180912 KLHDC2 Ecoli 01.18246 .18246 .2 \\
\hline & & FLFGG & $h$ & 540.2820 & 1 & - & - & 11.8808 & 180912 KLHDC2 Ecoli 01.11802.11802.1 \\
\hline & & TVGNRGFVFGG & $D$ & 555.7884 & 2 & 0.999999 & 1 & 0.3637 & 180912_KLHDC2 Ecoli_01.09887.09887.2 \\
\hline & & VGNRGFVFGG & $x$ & 505.2652 & 2 & 0.995331 & 0.8021 & 0.1754 & 180912_KLHDC2_Ecoli_01.09377.09377.2 \\
\hline & & NRGFVFGG & $r$ & 427.2200 & 2 & 0.999999 & 1 & 0.1230 & 180912_KLHDC2_Ecoli_01.08795.08795.2 \\
\hline & & FVFGG & $f$ & 526.2669 & 1 & - & - & 0.3150 & 180912 KLHDC2 Ecoli 01.10724.10724.1 \\
\hline & & HTACASDEGEVIVFGG & $U$ & 796.3599 & 2 & 0.997091 & 0.8334 & 0.0234 & 180912_KLHDC2_Ecoli_01.12223.12223.2 \\
\hline & & ACASDEGEVIVFGG & $K$ & 677.3041 & 2 & 0.999999 & 1 & 1.1273 & 180912_KLHDC2_Ecoli_01.15395.15395.2 \\
\hline & & CASDEGEVIVFGG & $H$ & 641.7860 & 2 & 0.999999 & 1 & 0.2641 & 180912 KLHDC2 Ecoli 01.15140 .15140 .2 \\
\hline & & ASDEGEVIVFGG & $F$ & 590.2795 & 2 & 0.999999 & 1 & 6.9175 & 180912_KLHDC2 Ecoli_ 01.14310 .14310 .2 \\
\hline & & SDEGEVIVFGG & $C$ & 554.7617 & 2 & 0.999999 & 1 & 4.0617 & 180912_KLHDC2_Ecoli_01.13952.13952.2 \\
\hline & & DEGEVIVFGG & $y$ & 511.2462 & 2 & 0.999999 & 1 & 0.8047 & 180912_KLHDC2_Ecoli_01.15036.15036.2 \\
\hline & & EGEVIVFGG & $t$ & 906.4572 & 1 & 0.999999 & 0.9992 & 3.8707 & 180912_KLHDC2_Ecoli_01.13658.13658.1 \\
\hline & & GEVIVFGG & $o$ & 777.4161 & 1 & 0.954359 & 0.5927 & 0.0000 & 180912_KLHDC2_Ecoli_01.11628.11628.1 \\
\hline & & EVIVFGG & $m$ & 720.3894 & 1 & - & - & 0.0264 & 180912 KLHDC2 Ecoli_01.12587.12587.1 \\
\hline & & VIVFGG & $j$ & 591.3505 & 1 & - & - & 0.1201 & 180912_KLHDC2_Ecoli_01.10301.10301.1 \\
\hline & & IVFGG & $c$ & 492.2821 & 1 & - & - & 68.3911 & 180912_KLHDC2_Ecoli_01.04861.04861.1 \\
\hline \multirow{8}{*}{ HIS-TSF } & \multirow{8}{*}{-} & AWSHPQFEKGGGSGGGSGG & $\mathrm{X}$ & 587.2652 & 3 & 0.999997 & 1 & 0.0000 & 180912 KLHDC2 Ecoli 01.04792 .04792 .3 \\
\hline & & WSHPQFEKGGGSGGGSGG & $\mathrm{W}$ & 563.5858 & 3 & 0.999992 & 0.2294 & 0.1204 & 180912_KLHDC2_Ecoli_01.04650.04650.3 \\
\hline & & SHPQFEKGGGSGGGSGG & $\mathrm{R}$ & 501.5594 & 3 & - & 0.9343 & - & 180912_KLHDC2_Ecoli_01.02643.02643.3 \\
\hline & & SSAWSHPQFEKGGGSGGG & $\mathrm{Y}$ & 578.261 & 3 & 0.999988 & 1 & 0.1259 & 180912_KLHDC2_Ecoli_01.04813.04813.3 \\
\hline & & AWSHPQFEKGGGSGGG & $\mathrm{T}$ & 520.2401 & 3 & 0.999999 & 1 & 0.0988 & 180912_KLHDC2_Ecoli_01.04290.04290.3 \\
\hline & & WSHPQFEKGGGSGGG & $\mathrm{Q}$ & 496.5613 & 3 & 0.999999 & 1 & 0.0845 & 180912_KLHDC2_Ecoli_01.04151.04151.3 \\
\hline & & SHPQFEKGGGSGGG & I & 434.5339 & 3 & 0.995331 & 0.8663 & 0.0175 & 180912_KLHDC2_Ecoli_01.02645.02645.3 \\
\hline & & HPQFEKGG & $\mathrm{s}$ & 450.2216 & 2 & 0.997091 & 0.3086 & 0.0011 & 180912_KLHDC2_Ecoli_01.02657.02657.2 \\
\hline UPF0441 protein YgiB & P0ADT2 & QRSATGTSSRSMGG & $N$ & 461.5543 & 3 & 0.99996 & 1 & 0.0008 & 180912 KLHDC2 Ecoli 01.02583 .02583 .3 \\
\hline \multirow{2}{*}{ Cell division protein FtsZ } & \multirow{2}{*}{ P0A9A6 } & TNDAVIKVIGVGGGG & $L$ & 678.876 & 2 & 0.661625 & 0.9626 & 0.0008 & 180912_KLHDC2_Ecoli_01.11554.11554.2 \\
\hline & & VIKVIGVGGGG & $v$ & 478.2983 & 2 & 0.999998 & 1 & - & 180912_KLHDC2_Ecoli_01.07675.07675.2 \\
\hline \multirow{2}{*}{ Glutamate dehydrogenase } & \multirow{2}{*}{ P00370 } & SSAIGPYKGG & $u$ & 468.7434 & 2 & 0.999992 & 1 & 0.0257 & 180912_KLHDC2_Ecoli_01.03704.03704.2 \\
\hline & & SAIGPYKGG & $q$ & 425.2275 & 2 & 0.999988 & 1 & 0.0245 & 180912_KLHDC2_Ecoli_01.03656.03656.2 \\
\hline \multirow{2}{*}{ Outer membrane protein $\mathrm{F}$} & \multirow{2}{*}{ P02931 } & SDDFFVGRVGG & $E$ & 578.2757 & 2 & 0.999997 & 1 & 0.0101 & 180912_KLHDC2_Ecoli_01.11706.11706.2 \\
\hline & & DDFFVGRVGG & $z$ & 534.7596 & 2 & 0.999997 & 1 & 0.0480 & 180912_KLHDC2_Ecoli_01.11891.11891.2 \\
\hline \multirow{2}{*}{ Malate dehydrogenase } & \multirow{2}{*}{ P61889 } & MKVAVLGAAGG & $w$ & 487.2795 & 2 & 0.9916 & 0.9997 & 0.0268 & 180912_KLHDC2_Ecoli_01.07840.07840.2 \\
\hline & & KVAVLGAAGG & $p$ & 421.7585 & 2 & 0.0666 & 0.6912 & 0.0021 & 180912_KLHDC2_Ecoli_01.04485.04485.2 \\
\hline Superoxide dismutase & P0AGD3 & TAFEGKSLEEIIRSSEGG & $Z$ & 637.9903 & 3 & 0.99996 & 1 & 0.0055 & 180912_KLHDC2_Ecoli_01.16997.16997.3 \\
\hline \multirow{2}{*}{$30 \mathrm{~S}$ ribosomal protein $\mathrm{S} 11$} & \multirow{2}{*}{ P0A7R9 } & TDRQGNALGWATAGG & $P$ & 737.8570 & 2 & 0.999992 & 1 & 0.0183 & 180912_KLHDC2_Ecoli_01.09082.09082.2 \\
\hline & & LGWATAGG & $n$ & 732.3681 & 1 & - & - & 0.1584 & 180912_KLHDC2_Ecoli_ 01.08650 .08650 .1 \\
\hline $30 \mathrm{~S}$ ribosomal protein $\mathrm{S} 12$ & P0A7S3 & IGGEGHNLQEHSVILIRGG & $\alpha$ & 497.2694 & 4 & 0.999962 & 1 & 0.0078 & 180912_KLHDC2_Ecoli_01.07766.07766.4 \\
\hline \multirow{2}{*}{ 30S ribosomal protein $\mathrm{S} 5$} & \multirow{2}{*}{ A0A140N6Z9 } & VFMQPASEGTGIIAGGA & $V$ & 803.4013 & 2 & 0.999999 & 1 & 0.0286 & 180912_KLHDC2_Ecoli_01.15328.15328.2 \\
\hline & & MQPASEGTGIIAGGA & $M$ & 680.3339 & 2 & 0.999998 & 0.9827 & 0.1135 & 180912_KLHDC2 Ecoli_01.08903.08903.2 \\
\hline \multirow{2}{*}{$\begin{array}{l}\text { Phage shock operon } \\
\text { rhodanese PspE }\end{array}$} & \multirow{2}{*}{ P23857 } & AEHWIDVRVPEQYQQEHVQGA & $\delta$ & 630.5578 & 4 & 0.999968 & 1 & 0.0102 & 180912_KLHDC2_Ecoli_01.11633.11633.4 \\
\hline & & IDVRVPEQYQQEHVQGA & $\beta$ & 666.0019 & 3 & 0.402979 & 0.9549 & 0.0232 & 180912_KLHDC2_Ecoli_01.08434.08434.3 \\
\hline
\end{tabular}




\begin{tabular}{|c|c|c|c|c|c|c|c|c|c|}
\hline $17 \mathrm{kDa}$ surface antigen & P0A905 & IQGGDDSNVIGAIGGA & $O$ & 722.3591 & 2 & 0.674402 & 0.9113 & 0.0246 & 180912_KLHDC2_Ecoli_01.12669.12669.2 \\
\hline $\begin{array}{l}\text { Phenylalanine--tRNA ligase } \\
\text { alpha subunit }\end{array}$ & P08312 & LRELPPEERPAAGA & $S$ & 502.6085 & 3 & 0.999963 & 1 & 0.0044 & 180912_KLHDC2_Ecoli_01.04031.04031.3 \\
\hline \multirow{2}{*}{$\begin{array}{l}\text { Histone family protein DNA- } \\
\text { binding protein }\end{array}$} & \multirow{2}{*}{ P0ACF4 } & KSQLIDKIAAGA & $G$ & 405.5750 & 3 & - & - & 0.0006 & 180912_KLHDC2_Ecoli_01.07687.07687.3 \\
\hline & & SQLIDKIAAGA & $B$ & 543.8123 & 2 & 0.991533 & 0.9996 & 0.0132 & 180912_KLHDC2_Ecoli_01.10066.10066.2 \\
\hline
\end{tabular}


Table S2. Peptide hydrophobicity values. From ThermoFisher Scientific Peptide Analyzing Tool Protein Peptide

KLHDC2

VSDGRHMFVWGG

ID Hydrophobicity

TVGNRGFVFGG

VGNRGFVFGG $h$

NRGFVFGG $\quad 23.5$

FVFGG $\quad x \quad 22.8$

$\begin{array}{lll}\text { SSDHLFLFGG } & r & 32.4\end{array}$

FLFGG $\quad f \quad 24.3$

\begin{tabular}{|c|c|c|c|}
\hline & ACASDEGEVIVFGG & $K$ & 26.6 \\
\hline & CASDEGEVIVFGG & $H$ & 27.2 \\
\hline & ASDEGEVIVFGG & $F$ & 26.4 \\
\hline & SDEGEVIVFGG & $C$ & 27.1 \\
\hline & DEGEVIVFGG & $y$ & 25.8 \\
\hline & EGEVIVFGG & $t$ & 26.2 \\
\hline & GEVIVFGG & $o$ & 26.3 \\
\hline & EVIVFGG & $m$ & 24.3 \\
\hline & VIVFGG & $j$ & 22.5 \\
\hline & IVFGG & $c$ & 16.3 \\
\hline \multirow{10}{*}{ His-TSF solubility tag } & AWSHPQFEKGGGSGGGSGG & $X$ & 17.9 \\
\hline & WSHPQFEKGGGSGGGSGG & $W$ & 17.1 \\
\hline & SHPQFEKGGGSGGGSGG & $R$ & 9.6 \\
\hline & SSAWSHPQFEKGGGSGGG & $Y$ & 19.9 \\
\hline & AWSHPQFEKGGGSGGG & $T$ & 18.7 \\
\hline & WSHPQFEKGGGSGGG & $Q$ & 17.9 \\
\hline & SHPQFEKGGGSGGG & $I$ & 9.9 \\
\hline & HPQFEKGG & $s$ & 11.0 \\
\hline & EKGGGSGGG & $l$ & 0.6 \\
\hline & GGGSGGG & $b$ & 0.9 \\
\hline UPF0441 protein YgiB & QRSATGTSSRSMGG & $N$ & 5.3 \\
\hline \multirow{2}{*}{ Cell division protein FtsZ } & TNDAVIKVIGVGGGG & $L$ & 25.6 \\
\hline & VIKVIGVGGGG & $v$ & 21.8 \\
\hline \multirow{2}{*}{ Glutamate dehydrogenase } & SSAIGPYKGG & $u$ & 12.8 \\
\hline & SAIGPYKGG & $q$ & 14.5 \\
\hline \multirow[b]{2}{*}{ Outer membrane protein $F$} & SDDFFVGRVGG & $E$ & 26.4 \\
\hline & DDFFVGRVGG & $z$ & 26.3 \\
\hline & MKVAVLGAAGG & $w$ & 24.0 \\
\hline Malate dehydrogenase & KVAVLGAAGG & $p$ & 20.4 \\
\hline Superoxide dismutase & TAFEGKSLEEIIRSSEGG & $Z$ & 31.5 \\
\hline \multirow{2}{*}{$30 \mathrm{~S}$ ribosomal protein $\mathrm{S} 11$} & TDRQGNALGWATAGG & $P$ & 22.5 \\
\hline & LGWATAGG & $n$ & 19.2 \\
\hline $30 \mathrm{~S}$ ribosomal protein $\mathrm{S} 12$ & IGGEGHNLQEHSVILIRGG & $\alpha$ & 31.1 \\
\hline \multirow{2}{*}{$30 \mathrm{~S}$ ribosomal protein $\mathrm{S} 5$} & VFMQPASEGTGIIAGGA & $\bar{V}$ & 32.2 \\
\hline & MQPASEGTGIIAGGA & $M$ & 22.8 \\
\hline \multirow{2}{*}{ Phage shock operon rhodanese PspE } & AEHWIDVRVPEQYQQEHVQGA & $\delta$ & 32.6 \\
\hline & IDVRVPEQYQQEHVQGA & $\beta$ & 23.9 \\
\hline 17 kDa surface antigen & IQGGDDSNVIGAIGGA & $O$ & 25.2 \\
\hline $\begin{array}{l}\text { Phenylalanine--tRNA ligase alpha } \\
\text { subunit }\end{array}$ & LRELPPEERPAAGA & $S$ & 21.1 \\
\hline \multirow{2}{*}{$\begin{array}{l}\text { Histone family protein DNA-binding } \\
\text { protein }\end{array}$} & KSQLIDKIAAGA & $\bar{G}$ & 22.2 \\
\hline & SQLIDKIAAGA & $B$ & 23.5 \\
\hline
\end{tabular}




\begin{tabular}{lrrr}
\hline & LVYGG & $e$ & 10.0 \\
& PEFGG & $d$ & 7.7 \\
& LPEFG & $k$ & 15.5 \\
Ambiguous origin & LIIGGG & $g$ & 16.4 \\
& LYFGG & $i$ & 19.1 \\
& LPPG & $a$ & 5.6
\end{tabular}


Table S3. Peptide location in E. coli protein of origin.

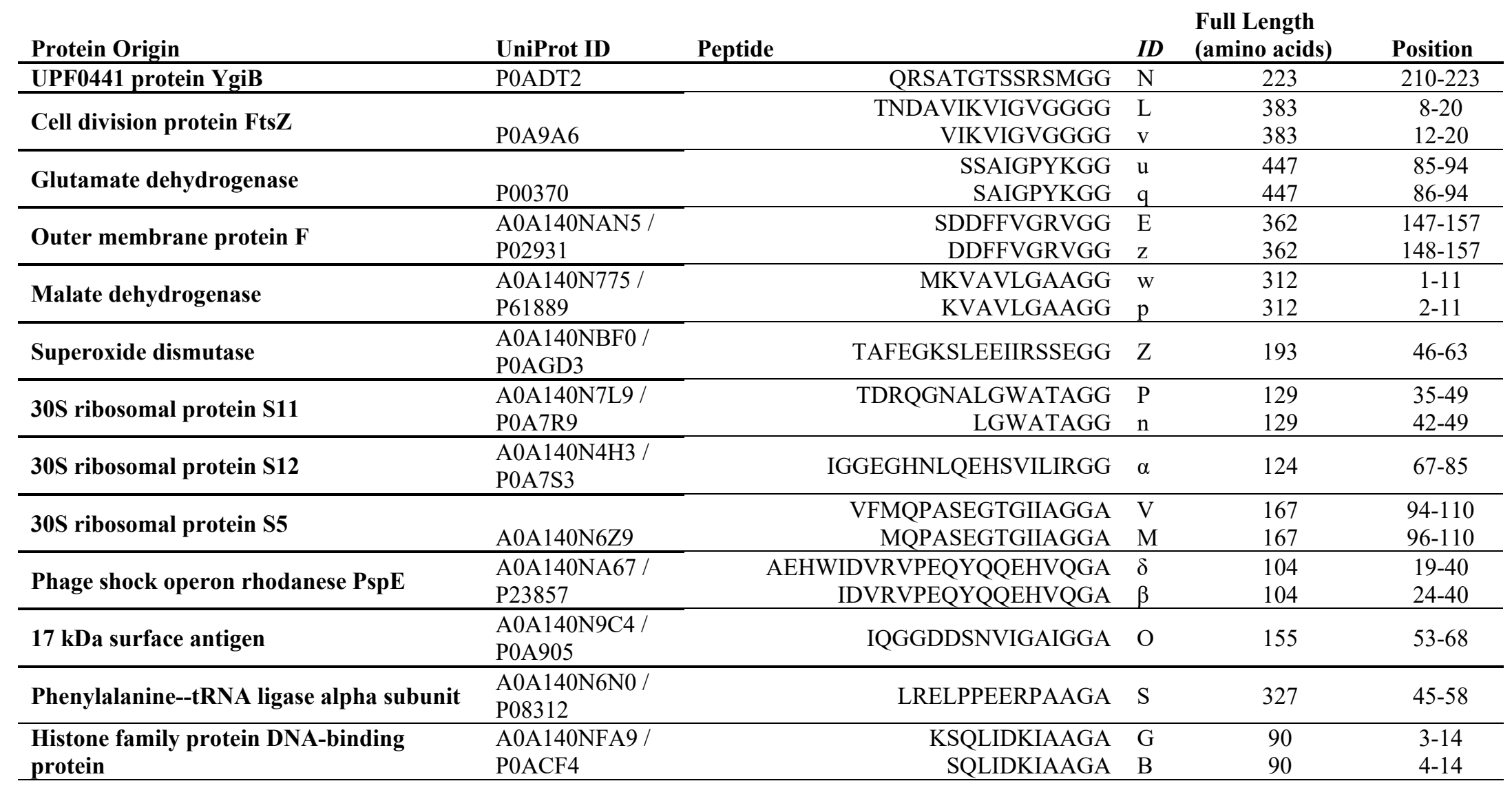




\section{Table S4. Potential Human Degrons}

\begin{tabular}{|c|c|c|c|}
\hline \multirow{2}{*}{ Potential Degron } & & Table S4: Exact degro & an proteins \\
\hline & Accession & Name & Alternative name \\
\hline FVWGG & Q96CH1.1 & Probable G-protein coupled receptor 146 & G-protein coupled receptor PGR8 \\
\hline FVFGG & Q9H2C0.1 & Gigaxonin & Kelch-like protein 16 \\
\hline FVFGG & Q9HOH3.1 & Kelch-like protein 25 & Ectoderm-neural cortex protein 2 \\
\hline FVFGG & Q6NT32.1 & Carboxylesterase 5A & Carboxylesterase-like urinary excreted protein homolog \\
\hline FVFGG & Q6UWW8.1 & Carboxylesterase 3 & Liver carboxylesterase 31 homolog \\
\hline FVFGG & Q7Z602.1 & Probable G-protein coupled receptor 141 & G-protein coupled receptor PGR13 \\
\hline FLFGG & Q8IWX8.3 & Calcium homeostasis endoplasmic reticulum protein & ERPROT 213-21 \\
\hline FLFGG & Q5XG92.2 & Carboxylesterase $4 \mathrm{~A}$ & \\
\hline FLFGG & Q02978.3 & Mitochondrial 2-oxoglutarate/malate carrier protein & OGCP \\
\hline FLFGG & Q8IYJ0.1 & PILR alpha-associated neural protein & PILR-associating neural protein \\
\hline FLFGG & Q9GZP9.1 & Derlin-2 & Degradation in endoplasmic reticulum protein 2 \\
\hline FLFGG & Q96Q80.2 & Derlin-3 & Degradation in endoplasmic reticulum protein 3 \\
\hline FLFGG & Q8IVM7.1 & Putative uncharacterized protein encoded by LINC00346 & \\
\hline FLFGG & Q9HC47.1 & Cutaneous T-cell lymphoma-associated antigen 1 & Protein cTAGE-1 \\
\hline FLFGG & Q7Z407.3 & CUB and sushi domain-containing protein 3 & CUB and sushi multiple domains protein 3 \\
\hline IVFGG & Q81ZF6.2 & Adhesion G-protein coupled receptor G4 & G-protein coupled receptor 112 \\
\hline IVFGG & Q6P3S6.1 & F-box only protein 42 & Just one F-box and Kelch domain-containing protein \\
\hline IVFGG & Q9H7R0.1 & Zinc finger protein 442 & \\
\hline RSMGG & P58107.3 & Epiplakin & $450 \mathrm{kDa}$ epidermal antigen \\
\hline RSMGG & P02787.3 & Serotransferrin & Transferrin \\
\hline RSMGG & Q8N543.1 & Prolyl 3-hydroxylase OGFOD1 & 2-oxoglutarate and iron-dependent oxygenase domain-containing protein 1 \\
\hline RSMGG & Q96125.1 & Splicing factor 45 & $45 \mathrm{kDa}$-splicing factor \\
\hline RSMGG & POCG43.1 & Putative protein FAM157C & \\
\hline RSMGG & POCG42.1 & Putative protein FAM157B & \\
\hline RSMGG & С9JC47.1 & Putative protein FAM157A & \\
\hline VGGGG & Q03164.5 & Histone-lysine $\mathrm{N}$-methyltransferase 2A & Lysine $\mathrm{N}$-methyltransferase $2 \mathrm{~A}$ \\
\hline VGGGG & Q562E7.2 & WD repeat-containing protein 81 & \\
\hline VGGGG & 095153.2 & Peripheral-type benzodiazepine receptor-associated protein 1 & PRAX-1 \\
\hline VGGGG & P29400.2 & Collagen alpha-5(IV) chain & \\
\hline VGGGG & Q5TGY3.1 & AT-hook DNA-binding motif-containing protein 1 & \\
\hline VGGGG & P49790.2 & Nuclear pore complex protein Nup153 & $153 \mathrm{kDa}$ nucleoporin \\
\hline VGGGG & 015014.2 & Zinc finger protein 609 & \\
\hline VGGGG & Q9H7P9.3 & Pleckstrin homology domain-containing family $\mathrm{G}$ member 2 & PH domain-containing family $\mathrm{G}$ member 2 \\
\hline VGGGG & 060503.4 & Adenylate cyclase type 9 & ATP pyrophosphate-lyase 9 \\
\hline VGGGG & P35568.1 & Insulin receptor substrate 1 & IRS-1 \\
\hline VGGGG & Q8TE59.2 & A disintegrin and metalloproteinase with thrombospondin motifs 19 & ADAM-TS 19 \\
\hline VGGGG & Q9BY07.2 & Electrogenic sodium bicarbonate cotransporter 4 & NBCe2 \\
\hline VGGGG & Q9Y4D2.3 & Sn1-specific diacylglycerol lipase alpha & DGL-alpha \\
\hline VGGGG & Q12860.1 & Contactin-1 & Glycoprotein gp135 \\
\hline VGGGG & Q96QC0.1 & Serine/threonine-protein phosphatase 1 regulatory subunit 10 & MHC class I region proline-rich protein CAT53 \\
\hline VGGGG & Q75VX8.3 & GRB2-associated and regulator of MAPK protein 2 & GRB2-associated and regulator of MAPK1-like \\
\hline VGGGG & Q68DC2.2 & Ankyrin repeat and SAM domain-containing protein 6 & Ankyrin repeat domain-containing protein 14 \\
\hline VGGGG & Q8TF30.2 & WASP homolog-associated protein with actin, membranes and microtubules & WAS protein homology region 2 domain-containing protein 1 \\
\hline VGGGG & P22059.1 & Oxysterol-binding protein 1 & \\
\hline VGGGG & Q96CN9.1 & GRIP and coiled-coil domain-containing protein 1 & Golgi coiled-coil protein 1 \\
\hline VGGGG & Q6P4Q7.3 & Metal transporter CNNM4 & Ancient conserved domain-containing protein 4 \\
\hline VGGGG & Q9C011.2 & Myotubularin-related protein 12 & Inactive phosphatidylinositol 3-phosphatase 12 \\
\hline VGGGG & Q9H4Q3.2 & PR domain zinc finger protein 13 & PR domain-containing protein 13 \\
\hline VGGGG & Q7L2J0.1 & 7SK snRNA methylphosphate capping enzyme & MePCE \\
\hline VGGGG & Q96F45.1 & Zinc finger protein 503 & \\
\hline VGGGG & 094826.1 & Mitochondrial import receptor subunit TOM70 & Mitochondrial precursor proteins import receptor \\
\hline VGGGG & Q8TE04.2 & Pantothenate kinase 1 & hPank \\
\hline VGGGG & Q9BQG1.1 & Synaptotagmin-3 & Synaptotagmin III \\
\hline VGGGG & Q16538.1 & Probable G-protein coupled receptor 162 & Gene-rich cluster gene A protein \\
\hline VGGGG & Q9H2S1.2 & Small conductance calcium-activated potassium channel protein 2 & SK2 \\
\hline VGGGG & Q96G74.1 & OTU domain-containing protein 5 & Deubiquitinating enzyme $\mathrm{A}$ \\
\hline VGGGG & Q9ULP9.2 & TBC1 domain family member 24 & \\
\hline VGGGG & P17342.2 & Atrial natriuretic peptide receptor 3 & Atrial natriuretic peptide clearance receptor \\
\hline VGGGG & Q8WUG5.1 & Solute carrier family 22 member 17 & $24 \mathrm{p} 3$ receptor \\
\hline VGGGG & Q9H598.2 & Vesicular inhibitory amino acid transporter & GABA and glycine transporter \\
\hline VGGGG & Q6ZRH9.1 & Uncharacterized protein FU46347 [Homo sapiens] & \\
\hline VGGGG & 014524.2 & Nuclear envelope integral membrane protein 1 & \\
\hline VGGGG & P14651.2 & Homeobox protein Hox-B3 & Homeobox protein Hox-2.7 \\
\hline VGGGG & P47901.1 & Vasopressin V1b receptor & V1bR \\
\hline VGGGG & Q2MJR0.1 & Sprouty-related, EVH1 domain-containing protein 3 & Spred-3 \\
\hline VGGGG & Q8TAD8.1 & Smad nuclear-interacting protein 1 & FHA domain-containing protein SNIP1 \\
\hline VGGGG & Q9NWY4.2 & Histone PARylation factor 1 & \\
\hline VGGGG & Q9GZT4.1 & Serine racemase & D-serine ammonia-lyase \\
\hline VGGGG & 060902.4 & Short stature homeobox protein 2 & Homeobox protein Og12X \\
\hline VGGGG & Q96GA7.1 & Serine dehydratase-like & L-serine deaminase \\
\hline VGGGG & P20132.2 & L-serine dehydratase/L-threonine deaminase & SDH \\
\hline VGGGG & Q63HQ0.1 & AP-1 complex-associated regulatory protein & $2 \mathrm{c} 18$ \\
\hline VGGGG & Q96H79.2 & Zinc finger $\mathrm{CCCH}$-type antiviral protein 1-like & \\
\hline VGGGG & P52945.1 & Pancreas/duodenum homeobox protein 1 & PDX-1 \\
\hline VGGGG & 075822.2 & Eukaryotic translation initiation factor 3 subunit J & elf3j \\
\hline VGGGG & Q8N7Y1.1 & Putative uncharacterized protein KIRREL3-AS3 & KIRREL3 antisense RNA 1 \\
\hline VGGGG & P10301.1 & Ras-related protein R-Ras & p23 \\
\hline VGGGG & Q9NV56.1 & MRG/MORF4L-binding protein & MRG-binding protein \\
\hline VGGGG & P62070.1 & Ras-related protein R-Ras2 & Ras-like protein TC21 \\
\hline VGGGG & Q961K1.2 & Biorientation of chromosomes in cell division protein 1 & Biorientation defective protein 1 \\
\hline VGGGG & Q9Y3D0.1 & Cytosolic iron-sulfur assembly component $2 B$ & MSS19-interacting protein of $18 \mathrm{kDa}$ \\
\hline PYKGG & Q99715.2 & Collagen alpha-1(XII) chain & \\
\hline PYKGG & Q13439.1 & Golgin subfamily A member 4 & $256 \mathrm{kDa}$ golgin \\
\hline PYKGG & Q05707.3 & Collagen alpha-1(XIV) chain & Undulin \\
\hline ATAGG & Q8IVF2.2 & Protein AHNAK2 & \\
\hline ATAGG & Q9P2D1.3 & Chromodomain-helicase-DNA-binding protein 7 & CHD-7 \\
\hline ATAGG & Q9Y4H2.2 & Insulin receptor substrate 2 & IRS-2 \\
\hline ATAGG & Q9ULL5.2 & Proline-rich protein 12 & \\
\hline ATAGG & Q6UXZ4.1 & Netrin receptor UNC5D & Protein unc- 5 homolog 4 \\
\hline ATAGG & Q8N196.3 & Homeobox protein SIX5 & DM locus-associated homeodomain protein \\
\hline ATAGG & & & \\
\hline
\end{tabular}




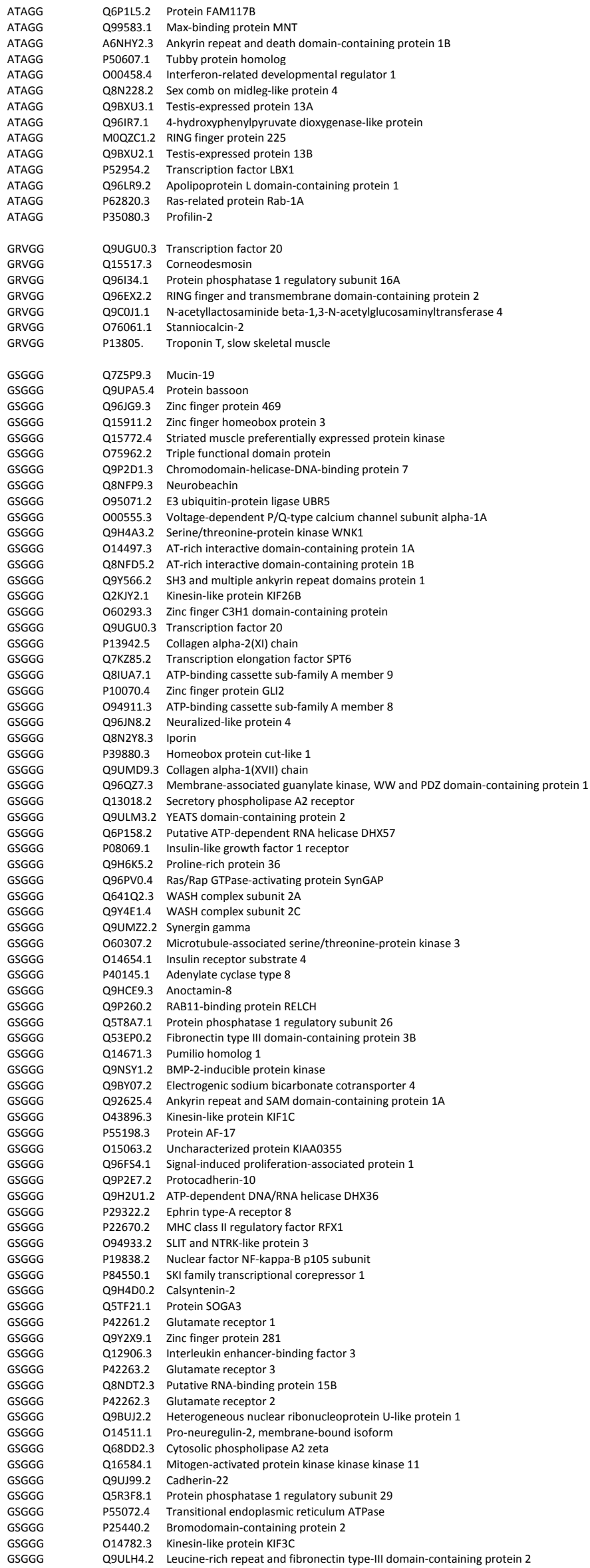

Amyotrophic lateral sclerosis 2 chromosomal region candidate gene 13 protein Class $\mathrm{D}$ basic helix-loop-helix protein 3

Nerve growth factor-inducible protein PC4

Glyoxalase domain-containing protein

Ladybird homeobox protein homolog

Vascular early response gene prote

YPT1-related protein

Profilin II

TCF-20

$S$ protein

Myosin phosphatase-targeting subunit 3

Transmembrane protein 118

UDP-GlcNAc:betaGal beta-1,3-N-acetylglucosaminyltransferase 4

STC-2

TnTs

MUC-19

Zinc finger protein 231

AT motif-binding factor 1

Aortic preferentially expressed protein 1

PTPRF-interacting protei

CHD-7

Lysosomal-trafficking regulator 2

E3 ubiquitin-protein ligase, HECT domain-containing 1

Brain calcium channel I

Erythrocyte $65 \mathrm{kDa}$ protein

ARID domain-containing protein $1 \mathrm{~A}$

ARID domain-containing protein $1 B$

Shank1

Coiled-coil domain-containing protein 131

TCF-20

hSPT6

GLI family zinc finger protein 2

Interacting protein of Rab1

CCAAT displacement protein

$180 \mathrm{kDa}$ bullous pemphigoid antigen 2

Atrophin-1-interacting protein 3

PLA2-R

DEAH box protein 57

Insulin-like growth factor I receptor

Neuronal RasGAP

Vaccinia virus penetration factor AP1 subunit gamma-binding protein 1

IRS-4

ATP pyrophosphate-lyase 8

Transmembrane protein $16 \mathrm{H}$

LisH domain and HEAT repeat-containing protein KIAA1468

Factor for adipocyte differentiation 104

HsPUM

NBCe2

Odin

ALL1-fused gene from chromosome 17 protein

Sipa-1

DEAD/H box polypeptide 36

$\mathrm{EPH}$ - and ELK-related kinase

Enhancer factor C

DNA-binding factor KBF1

Functional Smad-suppressing element on chromosome 15 Alcadein-gamma

GluR-1

GC-box-binding zinc finger protein 1

Double-stranded RNA-binding protein 76

GluR-3

One-twenty two protein 3

GluR-2

Adenovirus early region $1 \mathrm{~B}$-associated protein 5

Pro-NRG2

cPLA2-zeta

Mixed lineage kinase 3

Pituitary and brain cadherin

Extracellular leucine-rich repeat and fibronectin type III domain-containing protein 2

TER ATPase

027.1.1

Synaptic adhesion-like molecule 1 


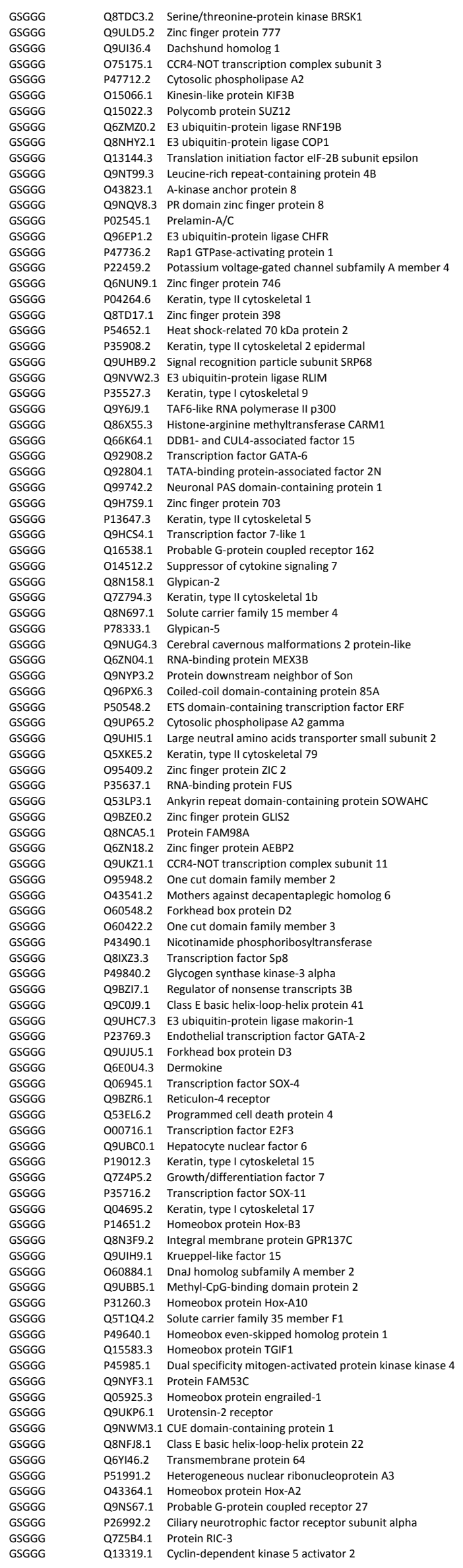

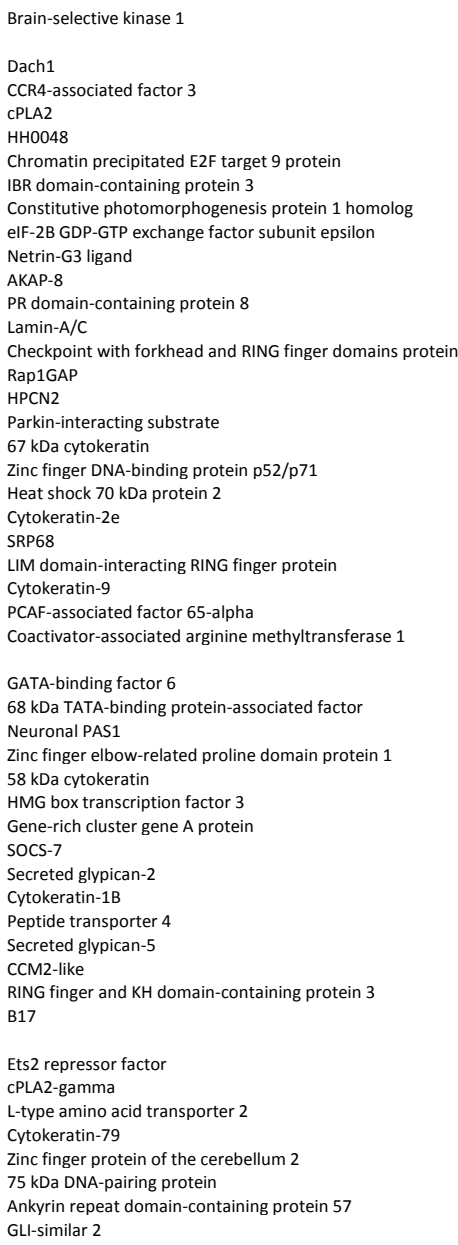

Adipocyte enhancer-binding protein 2

Hepatocyte nuclear factor 6-beta

MAD homolog 6

Forkhead-related protein FKHL17

One cut homeobox 3

NAmPRTase

Specificity protein

GSK-3 alpha

Nonsense mRNA reducing factor $3 \mathrm{~B}$

bHLHe41

RING finger protein 61

GATA-binding protein 2

HNF3/FH transcription factor genesis

Epidermis-specific secreted protein SK30/SK89

Nogo recept

E2F-3

Cytokeratin-15

GDF-7

Homeobox protein Hox-2.7

Transmembrane 7 superfamily member 1 -like 2 protein Kidney-enriched krueppel-like factor Cell cycle progression restoration gene 3 prote Demethylase

Homeobox protein Hox-1.8

EVX-1

5'-TG-3'-interacting factor 1

MAP kinase kinase 4

Homeobox protein en-1

UR-2-R

bHLHe22

hnRNPA3

Homeobox protein Hox-1K

Super conserved receptor expressed in brain 1

CNTF receptor subunit alpha

Resistant to inhibitor of cholinesterase 3

CDK5 activator 2 


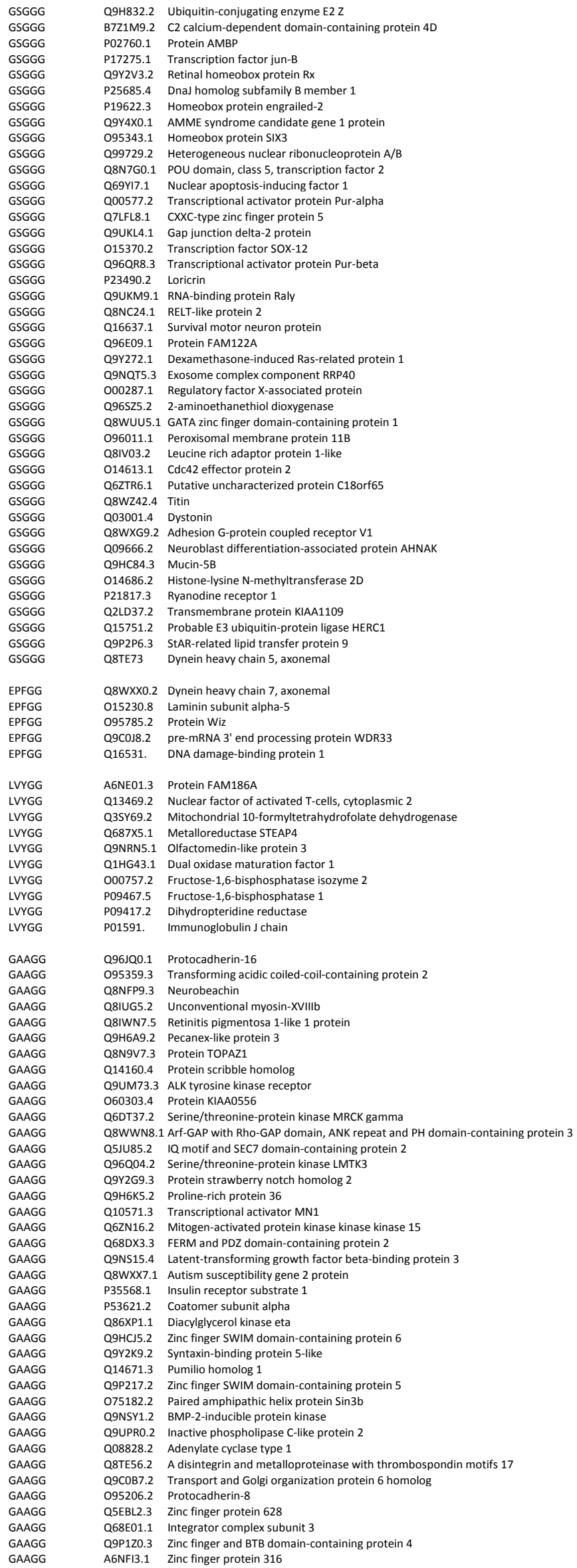

Alpha-1-microglobulin

Retina and anterior neural fold homeobox protein DnaJ protein homolog 1

Sine oculis homeobox homolog 3

$\operatorname{hnRNP} A / B$

Sperm 1 POU domain transcription factor

Puriner

Connexin-36

Protein SOX-22

Purine-rich element-binding protein B

Autoantigen $\mathrm{p} 542$

Component of gems 1

Activator of G-protein signaling 1

Exosome component 3

RFX-associated protein

Cysteamine dioxygenase

Ocular development-associated gene protein Peroxin-11B

Binder of Rho GTPases 1

Connectin

$230 \mathrm{kDa}$ bullous pemphigoid antigen

ADGRV 1

Desmoyokin

MUC-5

Lysine $\mathrm{N}$-methyltransferase 2D

RYR-1

Fragile site-associated protein

HECT domain and RCC1-like domain-containing protein 1 START domain-containing protein 9

Axonemal beta dynein heavy chain 5

Axonemal beta dynein heavy chain 7

Laminin-10 subunit alpha

Widely-interspaced zinc finger-containing protein

WD repeat-containing protein 33

DDB p127 subunit

NF-ATC2

Mitochondrial 10-FTHFDH

Six-transmembrane epithelial antigen of prostate 4

HNOEL-iso

Dual oxidase activator 1

FBPase 2

FBPase 1

HDHPR

Joining chain of multimeric IgA and IgM

Cadherin-19

Anti-Zuai-1

Lysosomal-trafficking regulator 2

Pecanex homolog protein 3

Testis- and ovary-specific PAZ domain-containing protein 1 Scribble

Anaplastic lymphoma kinase

CDC42-binding protein kinase gamma Centaurin-delta-3

Lemur tyrosine kinase 3

Probable tumor suppressor protein MN1

Apoptosis signal-regulating kinase 3

PDZ domain-containing protein 4

LTBP-3

IRS-1

Alpha-coat protein

DAG kinase eta

Lethal(2) giant larvae protein homolog 4

HsPUM

Histone deacetylase complex subunit $\operatorname{Sin} 3 b$

BIKe

PLC-L(2)

ATP pyrophosphate-lyase 1

ADAM-TS 17

Transmembrane and coiled-coil domain-containing protein 7 Arcadlin

Int3

KAISO-like zinc finger protein 1 


\begin{tabular}{|c|c|}
\hline GAAGG & Q6DN14.2 \\
\hline GAAGG & Q6UB35.1 \\
\hline GAAGG & Q9NRU3.3 \\
\hline GAAGG & P11586.3 \\
\hline GAAGG & Q9UMF0.3 \\
\hline GAAGG & 060502.2 \\
\hline GAAGG & Q9BXP2.1 \\
\hline GAAGG & Q00653.4 \\
\hline GAAGG & P30530.4 \\
\hline GAAGG & Q9UL51.3 \\
\hline GAAGG & Q $75 \mathrm{~V} \times 8.3$ \\
\hline GAAGG & 043525.2 \\
\hline GAAGG & P29376.3 \\
\hline GAAGG & P42226.1 \\
\hline GAAGG & Q96PV6.3 \\
\hline GAAGG & Q13033.3 \\
\hline GAAGG & Q9BQ17.3 \\
\hline GAAGG & Q08AE8.3 \\
\hline GAAGG & P85037.1 \\
\hline GAAGG & Q96NY7.3 \\
\hline GAAGG & Q9NQV8.3 \\
\hline GAAGG & Q2M3M2.2 \\
\hline GAAGG & 043524.1 \\
\hline GAAGG & 060299.1 \\
\hline GAAGG & Q8NFH8.2 \\
\hline GAAGG & P35908.2 \\
\hline GAAGG & 095935.3 \\
\hline GAAGG & P30154.3 \\
\hline GAAGG & AOPJK1.2 \\
\hline GAAGG & Q92908.2 \\
\hline GAAGG & P14866.2 \\
\hline GAAGG & Q9HCS4.1 \\
\hline GAAGG & Q5GH72.1 \\
\hline GAAGG & P19440.2 \\
\hline GAAGG & P36268.3 \\
\hline GAAGG & A6NGU5.2 \\
\hline GAAGG & Q12948.3 \\
\hline GAAGG & P50991.4 \\
\hline GAAGG & Q96EG3.2 \\
\hline GAAGG & Q9Y4C5.2 \\
\hline GAAGG & Q9BZE0.2 \\
\hline GAAGG & Q13596.3 \\
\hline GAAGG & Q6VOL0.2 \\
\hline GAAGG & |3L273.1 \\
\hline GAAGG & Q81XS0.2 \\
\hline GAAGG & Q13064.1 \\
\hline GAAGG & Q8NCB2.2 \\
\hline GAAGG & Q99958.1 \\
\hline GAAGG & P78415.3 \\
\hline GAAGG & Q9UL49.2 \\
\hline GAAGG & Q9UNW9.1 \\
\hline GAAGG & P30532.2 \\
\hline GAAGG & Q16676.1 \\
\hline GAAGG & B1AL88.1 \\
\hline GAAGG & Q14849.2 \\
\hline GAAGG & Q12947.2 \\
\hline GAAGG & Q9ULK6.2 \\
\hline GAAGG & 015105.1 \\
\hline GAAGG & Q8WXS5.3 \\
\hline GAAGG & Q86SH2.1 \\
\hline GAAGG & P25490.2 \\
\hline SSEGG & Q96RW7.2 \\
\hline SSEGG & Q9UPA5.4 \\
\hline SSEGG & Q15772.4 \\
\hline SSEGG & Q9Y566.2 \\
\hline SSEGG & P49815.2 \\
\hline SSEGG & Q9Y6Y1.4 \\
\hline SSEGG & Q9UPX8.3 \\
\hline SSEGG & Q76176.1 \\
\hline SSEGG & Q9Y4H2.2 \\
\hline SSEGG & P12821.1 \\
\hline SSEGG & Q9HCE3.2 \\
\hline SSEGG & 094854.4 \\
\hline SSEGG & Q9P218.4 \\
\hline SSEGG & P54098.1 \\
\hline SSEGG & 015068.2 \\
\hline SSEGG & Q.5QJ38.1 \\
\hline SSEGG & Q9P2N6.2 \\
\hline SSEGG & P52630.1 \\
\hline SSEGG & Q16620.1 \\
\hline SSEGG & Q8WUQ7.3 \\
\hline SSEGG & Q6ZRP7.3 \\
\hline SSEGG & Q5TEJ8.1 \\
\hline SSEGG & P56182.1 \\
\hline SSEGG & 015143.3 \\
\hline SSEGG & Q12799.3 \\
\hline SSEGG & B9ZVM9.2 \\
\hline SSEGG & Q86SK9.2 \\
\hline SSEGG & Q9BQ89.1 \\
\hline SSEGG & Q9Y3E1.1 \\
\hline SSEGG & Q9UHA7.1 \\
\hline SSEGG & Q5TCM9.1 \\
\hline SSEGG & Q5TA78.1 \\
\hline SSEGG & Q5TA77.1 \\
\hline SSEGG & Q9BYE3.1 \\
\hline SSEGG & Q.5T5B0.1 \\
\hline LIRGG & P29475.2 \\
\hline
\end{tabular}




\begin{tabular}{|c|c|c|c|}
\hline LIRGG & P13671.3 & Complement component $\mathrm{C} 6$ & \\
\hline LIRGG & Q13330.2 & Metastasis-associated protein MTA1 & \\
\hline LIRGG & 014531.2 & Dihydropyrimidinase-related protein 4 & DRP-4 \\
\hline LIRGG & P02748.2 & Complement component $\mathrm{C} 9$ & Complement component C9a \\
\hline LIRGG & Q14117.1 & Dihydropyrimidinase & DHP \\
\hline LIRGG & Q9H765.1 & Ankyrin repeat and SOCS box protein 8 & ASB-8 \\
\hline LIRGG & Q86SZ2.1 & Trafficking protein particle complex subunit $6 B$ & TRAPP complex subunit 6B \\
\hline LIRGG & B1AK76.2 & Putative SNURF-like protein & \\
\hline AIGGA & Q5VU43.3 & Myomegalin & Cardiomyopathy-associated protein 2 \\
\hline AIGGA & Q8WWN8.1 & Arf-GAP with Rho-GAP domain, ANK repeat and PH domain-containing protein 3 & Centaurin-delta-3 \\
\hline AIGGA & Q9UBN7.2 & Histone deacetylase 6 & HD6 \\
\hline AIGGA & Q13423.3 & $N A D(P)$ transhydrogenase, mitochondrial & Nicotinamide nucleotide transhydrogenase \\
\hline AIGGA & P48163.1 & NADP-dependent malic enzyme & NADP-ME \\
\hline AIGGA & Q8WWR8.3 & Sialidase-4 & $\mathrm{N}$-acetyl-alpha-neuraminidase 4 \\
\hline AIGGA & P35998.3 & 265 proteasome regulatory subunit 7 & $26 \mathrm{~S}$ proteasome AAA-ATPase subunit RPT1 \\
\hline AIGGA & Q9BY41.2 & Histone deacetylase 8 & HD8 \\
\hline AIGGA & P20138.2 & Myeloid cell surface antigen CD33 & Sialic acid-binding Ig-like lectin 3 \\
\hline AIGGA & Q9H1K6.1 & Talin rod domain-containing protein 1 & Mesoderm development candidate 1 \\
\hline AIGGA & Q9NQN1.2 & Olfactory receptor $2 \mathrm{~S} 2$ & Olfactory receptor OR9-3 \\
\hline AIGGA & 075880.1 & Protein SCO1 homolog, mitochondrial & \\
\hline PAAGA & P21817.3 & Ryanodine receptor 1 & RYR-1 \\
\hline PAAGA & Q9NYC9.3 & Dynein heavy chain 9 , axonemal & Axonemal beta dynein heavy chain 9 \\
\hline PAAGA & Q9UPA5.4 & Protein bassoon & Zinc finger protein 231 \\
\hline PAAGA & Q15772.4 & Striated muscle preferentially expressed protein kinase & Aortic preferentially expressed protein 1 \\
\hline PAAGA & Q9UM47.2 & Neurogenic locus notch homolog protein 3 & Notch 3 \\
\hline PAAGA & Q9Y4D7.3 & Plexin-D1 & \\
\hline PAAGA & 043151.3 & Methylcytosine dioxygenase TET3 & \\
\hline PAAGA & 060312.2 & Probable phospholipid-transporting ATPase VA & ATPase class $V$ type $10 \mathrm{~A}$ \\
\hline PAAGA & Q9UMD9.3 & Collagen alpha-1(XVII) chain & $180 \mathrm{kDa}$ bullous pemphigoid antigen 2 \\
\hline PAAGA & Q5JU85.2 & IQ motif and SEC7 domain-containing protein 2 & \\
\hline PAAGA & P41594.2 & Metabotropic glutamate receptor 5 & mGluR5 \\
\hline PAAGA & Q9UNS1.2 & Protein timeless homolog & hTIM \\
\hline PAAGA & Q9NQC3.2 & Reticulon-4 & Foocen \\
\hline PAAGA & Q9NZJ5.3 & Eukaryotic translation initiation factor 2-alpha kinase 3 & PRKR-like endoplasmic reticulum kinase \\
\hline PAAGA & Q93073.3 & Selenocysteine insertion sequence-binding protein 2-like & SECIS-binding protein 2-like \\
\hline PAAGA & 094898.3 & Leucine-rich repeats and immunoglobulin-like domains protein 2 & LIG-2 \\
\hline PAAGA & Q5JWF2.2 & Guanine nucleotide-binding protein $\mathrm{G}(\mathrm{s})$ subunit alpha isoforms XLas & Adenylate cyclase-stimulating $\mathrm{G}$ alpha protein \\
\hline PAAGA & Q58A45.3 & PAN2-PAN3 deadenylation complex subunit PAN3 & PAB1P-dependent poly(A)-specific ribonuclease \\
\hline PAAGA & Q96DT7.2 & Zinc finger and BTB domain-containing protein 10 & Zinc finger protein RIN ZF \\
\hline PAAGA & Q96RN5.2 & Mediator of RNA polymerase II transcription subunit 15 & Activator-recruited cofactor $105 \mathrm{kDa}$ component \\
\hline PAAGA & Q9P1Z3.2 & Potassium/sodium hyperpolarization-activated cyclic nucleotide-gated channel 3 & \\
\hline PAAGA & Q15059.1 & Bromodomain-containing protein 3 & RING3-like protein \\
\hline PAAGA & Q96ED9.3 & Protein Hook homolog 2 & h-hook2 \\
\hline PAAGA & А9YTQ3.3 & Aryl hydrocarbon receptor repressor & AhR repressor \\
\hline PAAGA & Q9UI46.1 & Dynein intermediate chain 1 , axonemal & Axonemal dynein intermediate chain 1 \\
\hline PAAGA & Q03169.2 & Tumor necrosis factor alpha-induced protein 2 & TNF alpha-induced protein 2 \\
\hline PAAGA & Q86XN8.3 & RNA-binding protein MEX3D & RING finger and $\mathrm{KH}$ domain-containing protein 1 \\
\hline PAAGA & Q13202.2 & Dual specificity protein phosphatase 8 & Dual specificity protein phosphatase $\mathrm{hVH}-5$ \\
\hline PAAGA & Q96K58.3 & Zinc finger protein 668 & \\
\hline PAAGA & 000499.1 & Myc box-dependent-interacting protein 1 & Amphiphysin II \\
\hline PAAGA & P21589.1 & $5^{\prime}$-nucleotidase & 5 -NT \\
\hline PAAGA & Q96FN4.3 & Copine-2 & Copine II \\
\hline PAAGA & Q86VQ1.1 & Glucocorticoid-induced transcript 1 protein & \\
\hline PAAGA & Q6P177.1 & Protein BHLHb9 & bHLHb9 \\
\hline PAAGA & P00390.2 & Glutathione reductase, mitochondrial & GR \\
\hline PAAGA & P78413.2 & Iroquois-class homeodomain protein IRX-4 & Homeodomain protein IRXA3 \\
\hline PAAGA & Q18PE1.1 & Protein Dok-7 & Downstream of tyrosine kinase 7 \\
\hline PAAGA & Q5VZB9.1 & Doublesex- and mab-3-related transcription factor A1 & \\
\hline PAAGA & Q865X3.2 & Tubulin epsilon and delta complex protein 1 & \\
\hline PAAGA & Q6NUT3.2 & Major facilitator superfamily domain-containing protein 12 & \\
\hline PAAGA & Q16676.1 & Forkhead box protein D1 & Forkhead-related protein FKHL8 \\
\hline PAAGA & Q03052.3 & POU domain, class 3 , transcription factor 1 & Octamer-binding protein 6 \\
\hline PAAGA & 015105.1 & Mothers against decapentaplegic homolog 7 & MAD homolog 7 \\
\hline PAAGA & P21917.3 & $\mathrm{D}(4)$ dopamine receptor & $\mathrm{D}(2 \mathrm{C})$ dopamine receptor \\
\hline PAAGA & Q995X4.1 & Photoreceptor-specific nuclear receptor & Nuclear receptor subfamily 2 group E member 3 \\
\hline PAAGA & Q14183.5 & Double C2-like domain-containing protein alpha & Doc2 \\
\hline PAAGA & 043435.1 & T-box transcription factor TBX1 & T-box protein 1 \\
\hline PAAGA & Q9Y6U7.2 & RING finger protein 215 & \\
\hline PAAGA & Q9Uנ37.1 & Alpha-N-acetylgalactosaminide alpha-2,6-sialyltransferase 2 & GalNAc alpha-2,6-sialyltransferase II \\
\hline PAAGA & Q9BUN1.1 & Protein MENT & Methylated in normal thymocytes protein \\
\hline PAAGA & Q99946.2 & Proline-rich transmembrane protein 1 & Dispanin subfamily D member 1 \\
\hline PAAGA & Q9UJA2.1 & Cardiolipin synthase (CMP-forming) & CLS \\
\hline PAAGA & Q96M98.2 & Parkin coregulated gene protein & Molecular chaperone/chaperonin-binding protein \\
\hline PAAGA & Q03014.1 & Hematopoietically-expressed homeobox protein HHEX & Homeobox protein HEX \\
\hline PAAGA & Q8NB15.2 & Zinc finger protein 511 & \\
\hline PAAGA & Q6MZM9.1 & Proline-rich protein 27 & \\
\hline PAAGA & 075340.1 & Programmed cell death protein 6 & Apoptosis-linked gene 2 protein homolog \\
\hline PAAGA & Q4GOI0.1 & Protein CCSMST1 & \\
\hline PAAGA & Q6UXT8.1 & ALK and LTK ligand 1 & Augmentor beta \\
\hline PAAGA & P05386.1 & 60 S acidic ribosomal protein $P 1$ & Large ribosomal subunit protein P1 \\
\hline IAAGA & 000468.6 & Agrin & Agrin $\mathrm{N}$-terminal $110 \mathrm{kDa}$ subunit \\
\hline IAAGA & Q6UB98.3 & Ankyrin repeat domain-containing protein 12 & Ankyrin repeat-containing cofactor 2 \\
\hline IAAGA & A8K8P3.2 & Protein SFI1 homolog & hSFI1 \\
\hline IAAGA & Q8NET8.2 & Transient receptor potential cation channel subfamily $V$ member 3 & TrpV3 \\
\hline IAAGA & 094956.2 & Solute carrier organic anion transporter family member $2 \mathrm{~B} 1$ & Organic anion transporter B \\
\hline IAAGA & 043246.3 & Cationic amino acid transporter 4 & CAT-4 \\
\hline IAAGA & P14635.1 & G2/mitotic-specific cyclin-B1 & \\
\hline IAAGA & Q3YBM2.2 & Transmembrane protein $176 \mathrm{~B}$ & Protein LR8 \\
\hline IAAGA & A6NJS3.1 & Putative V-set and immunoglobulin domain-containing-like protein IGHV1OR21-1 & Immunoglobulin heavy variable 1 orphon 21-1 \\
\hline IPPGG & Q9UPA5.4 & Protein bassoon & Zinc finger protein 231 \\
\hline IPPGG & Q9NYQ6.1 & Cadherin EGF LAG seven-pass G-type receptor 1 & Cadherin family member 9 \\
\hline IPPGG & Q7Z7M0.2 & Multiple epidermal growth factor-like domains protein 8 & Multiple EGF-like domains protein 8 \\
\hline IPPGG & Q9ULT8.3 & E3 ubiquitin-protein ligase HECTD1 & E3 ligase for inhibin receptor \\
\hline IPPGG & 043497.3 & Voltage-dependent T-type calcium channel subunit alpha- $1 \mathrm{G}$ & Cav3.1c \\
\hline IPPGG & Q8IZY2.3 & Phospholipid-transporting ATPase ABCA7 & ABCA-SSN \\
\hline
\end{tabular}


IPPGG Q86UU0.1 B-cell CLL/lymphoma 9-like protein

IPPGG

IPPGG

IPPGG

IPPGG

IPPGG

IPPGG

IPPGG

IPPGG

IPPGG

IPPGG

IPPGG

IPPGG

IPPGG

IPPGG

IPPGG

IPPGG

IPPGG

IPPGG

PPGG

IPPGG

IPPGG

IPPGG

IPPGG

IPPGG

IPPGG

IPPGG

IPPGG

IPPGG

IPPGG

IPPGG

IPPGG

PGG

IPPGG

IPPGG

IPPGG

IPPGG

IPPGG

IPPGG

PEFGG

PEFGG

PEFGG

PEFGG

PEFGG

PEFGG

IIGGG

IIGGG

IIGGG

IIGGG

IIGGG

IIGGG

IIGGG

IIGGG

IIGGG

LYFGG

LYFGG

LYFGG

Q5T5C0.1 Syntaxin-binding protein 5

Q13608.2 Peroxisome assembly factor 2

Q86TD4.2 Sarcalumenin

Q7Z6J0.2 E3 ubiquitin-protein ligase SH3RF1

Q9Y2T1.1 Axin-2

A8K0R7.1 Zinc finger protein 839

095936.3 Eomesodermin homolos

Q12889.1 Oviduct-specific glycoprotein

Q5SNT2.1 Transmembrane protein 201

A6NMB9.2 Putative fidgetin-like protein 2

Q96JJ6.2 Junctophilin-4

09Y6J9.1 TAF6-like RNA polymerase II p300

Q9ULZ9.4 Matrix metalloproteinase-17

Q9HCS4.1 Transcription factor 7-like 1

000400.1 Acetyl-coenzyme A transporter 1

Q14117.1 Dihydropyrimidinase

Q400G9.1 Archaemetzincin-1

075688.1 Protein phosphatase 1B

P41225.2 Transcription factor SOX-3

Q8N819.2 Probable protein phosphatase 1N

Q9H2A9.2 Carbohydrate sulfotransferase 8

Q92949.3 Forkhead box protein J1

P31391.2 Somatostatin receptor type 4

35813.1 Protein phosphatase $1 \mathrm{~A}$

015391.1 Transcription factor YY2

Q96MM3.2 Zinc finger protein 42 homolos

Q9UBV8.1 Peflin

P0C2L3.1 Protein FAM163B

Q9Y4H4.1 G-protein-signaling modulator 3

Q8WZ42.4 Titin

P55011.1 Solute carrier family 12 member 2

Q9UHW9.2 Solute carrier family 12 member 6

Q9H2X9.3 Solute carrier family 12 member 5

Q13621.2 Solute carrier family 12 member 1

Q9UP95.2 Solute carrier family 12 member 4

Q9Y666.3 Solute carrier family 12 member 7

Q96T83.1 Sodium/hydrogen exchanger 7

Q86VQ6.4 Thioredoxin reductase 3

Q9UJU5.1 Forkhead box protein D3

P00558.3 Phosphoglycerate kinase 1

P07205.3 Phosphoglycerate kinase 2

P19623.1 Spermidine synthase

P30301.1 Lens fiber major intrinsic protein

096015.1 Dynein light chain 4, axonemal
060244.2 Mediator of RNA polymerase II transcription subunit 14

Q8TD43.1 Transient receptor potential cation channel subfamily M member 4

Q9Y4D1.2 Disheveled-associated activator of morphogenesis 1

Q86T65.3 Disheveled-associated activator of morphogenesis 2

Q9H8H2.2 Probable ATP-dependent RNA helicase DDX31

015033.3 Apoptosis-resistant E3 ubiquitin protein ligase 1

P51178.2 1-phosphatidylinositol 4,5-bisphosphate phosphodiesterase delta-1

Q8NE28.5 Serine/threonine kinase-like domain-containing protein STKLD1

Q16822.4 Phosphoenolpyruvate carboxykinase

Q6UUV9.2 CREB-regulated transcription coactivator 1

Q7L9B9.2 Endonuclease/exonuclease/phosphatase family domain-containing protein 1

Q5TF58.3 Intermediate filament family orphan 2

043516.3 WAS/WASL-interacting protein family member 1

P25490.2 Transcriptional repressor protein YY1

Q9BYG4.1 Partitioning defective 6 homolog gamma

Q8WZ04.3 Transmembrane O-methyltransferase

A8MU10.1 Putative uncharacterized protein ENSP00000381562

Q8N4T4.1 Rho guanine nucleotide exchange factor 39

Q16881.3 Thioredoxin reductase 1, cytoplasmic

095831.1 Apoptosis-inducing factor 1, mitochondrial

Q6NXP6.2 NADP-dependent oxidoreductase domain-containing protein 1

Q99437.1 V-type proton ATPase $21 \mathrm{kDa}$ proteolipid subunit

Q6N063.2 2-oxoglutarate and iron-dependent oxygenase domain-containing protein 2

Q9UNI6.1 Dual specificity protein phosphatase 12
B-cell lymphoma 9-like protein

Activator-recruited cofactor $150 \mathrm{kDa}$ component

hTRPM4

Lethal(2) giant larvae protein homolog 3

PAF-2

Plenty of SH3s

DEAD box protein 31

Axin-like protein

Apoptosis-resistant HECT-type E3 ubiquitin transferase 1

Renal carcinoma antigen NY-REN-50

Phosphoinositide phospholipase C-delta-1

T-box brain protein 2

Serine/threonine kinase-like domain-containing protein 1

Estrogen-dependent oviduct protein

Spindle-associated membrane protein 1

PEPCK-M

Mucoepidermoid carcinoma translocated protein 1

JP-4

PCAF-associated factor 65-alpha

MMP-17

HMG box transcription factor 3

AT-1

DHP

Protein PRPL-2

Archeobacterial metalloproteinase-like protein 1

Protein phosphatase $2 \mathrm{C}$ isoform beta

GalNAc-4-O-sulfotransferase 1

Forkhead-related protein FKHL13

Delta transcription factor

SS-4-R

Protein phosphatase $2 \mathrm{C}$ isoform alpha

PAR-6 gamma

Yin and yang 2

Zfp-42

Catechol O-methyltransferase 2

PEF protein with a long $\mathrm{N}$-terminal hydrophobic domain

Activator of G-protein signaling 4

Connectin

Basolateral Na-K-Cl symporte

Electroneutral potassium-chloride cotransporter 3

Electroneutral potassium-chloride cotransporter 2

Bumetanide-sensitive sodium-(potassium)-chloride cotransporter 2

Electroneutral potassium-chloride cotransporter 1

Electroneutral potassium-chloride cotransporter 4

$\mathrm{Na}(+) / H(+)$ exchanger 7

TR

Thioredoxin and glutathione reductase

Programmed cell death protein 8

HNF3/FH transcription factor genesis

Cell migration-inducing gene 10 protein

Phosphoglycerate kinase, testis specific

Pyrroline-5-carboxylate reductase-like protein C14orf148

SPDSY

Aquaporin-0

V-ATPase $21 \mathrm{kDa}$ proteolipid subunit

Dual specificity tyrosine phosphatase YVH1 


\section{References}

(1) Wang, M.; Herrmann, C. J.; Simonovic, M.; Szklarczyk, D.; von Mering, C. Version 4.0 of PaxDb: Protein Abundance Data, Integrated across Model Organisms, Tissues, and CellLines. PROTEOMICS 2015, 15 (18), 3163-3168. https://doi.org/10.1002/pmic.201400441. 


\title{
Appendix: Determining Relative Abundances
}

\author{
In [1]: import numpy as np \\ import scipy \\ from scipy import optimize \\ from scipy.interpolate import interp1d \\ from scipy.optimize import minimize \\ import matplotlib as $\mathrm{mpl}$ \\ import matplotlib.pyplot as plt \\ from matplotlib import $\mathrm{cm}$ \\ \%matplotlib inline
}

\section{Loading experimental data}

In [14]: $\mathrm{dt}=\mathrm{np}$. loadtxt('SI_12+_experiment.txt') \#Experimental Data for 12+ charge sta te

$\mathrm{x}=\mathrm{dt}[:, 0] \# \mathrm{~m} / \mathrm{z}$ values

$\mathrm{y}=\mathrm{dt}[:, 1]$ \#intensity

\#Resampling $\mathrm{m} / \mathrm{z}$ axis $(x)$ with 4 points between each $\mathrm{m} / \mathrm{z}$ value

$f=\operatorname{interp} 1 d(x, y)$

$\mathrm{x} 2=\mathrm{np}$.linspace $(3190,3475,1140)$

y2 $=f(x 2)$ \#Using resampled $m / z$ values to obtain y2 values

\section{Creating the feature used to estimate peptide abundance}

In [15]: \#The apo feature (peak shape of the KLHDC2) is used as a template, \#and is extracted from the experimental data:

x_shield $=(3270>=x 2) \&(x 2>=3190)$ \#These are the $\mathrm{m} / \mathrm{z}$ bounds for the apo $f$ eature

ymodel $=\left(x_{\text {_s shield }} * 1\right) * \mathrm{y} 2$ \#ymodel= intesity values in the $\mathrm{m} / \mathrm{z}$ range of the ap o peak

np.savetxt('SI_Ymodel_12+.txt', ymodel) \#saving the apo feature to a text file 
In [16]: \#plotting the apo feature and the experimental data

Ymodel = np.loadtxt('SI_Ymodel_12+.txt') \#The apo feature used for modeling

plt.plot $(\mathrm{x} 2, \mathrm{y} 2)$ \#experimental data, blue

plt.plot(x2,Ymodel) \#Apo feature, orange

Out[16]: [<matplotlib.lines.Line2D at 0x3bc103b198>]

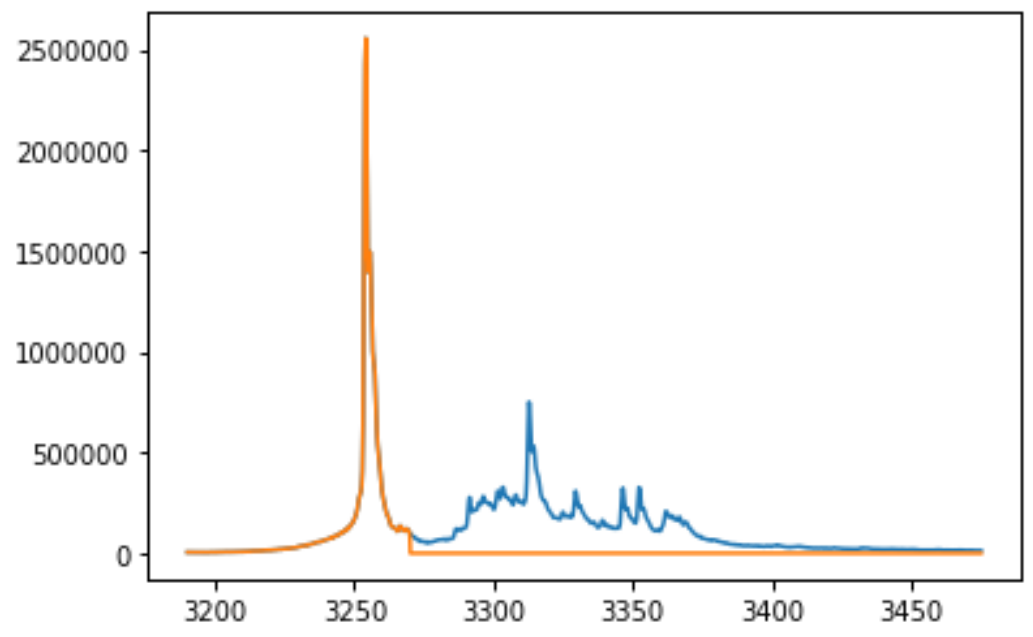

In [23]: \#Since the tailing edge of the feature was obscured by the peptide-bound porti on

\#of the spectrum, an artificial tail was added to the apo feature. The leading \#edge of the feature (from approx. 3190 to $3240 \mathrm{~m} / \mathrm{z}$ ) was copied, reversed, and a dded

\#to the apo feature starting at approximately $3270 \mathrm{~m} / \mathrm{z}$. The intensity values o $f$

\#the added tail were reduced to bring the values closer to the baseline.

YMODEL $=$ np.loadtxt ('SI_Ymodel_12+_tail.txt')

plt.plot $(\mathrm{x} 2, \mathrm{y} 2)$ \#experimental data, blue

plt.plot(x2, YMODEL) \#Apo feature, orange

\section{4}

\section{Out [23]: [<matplotlib.lines.Line2D at $0 \times 3$ bc126bdd8>]}

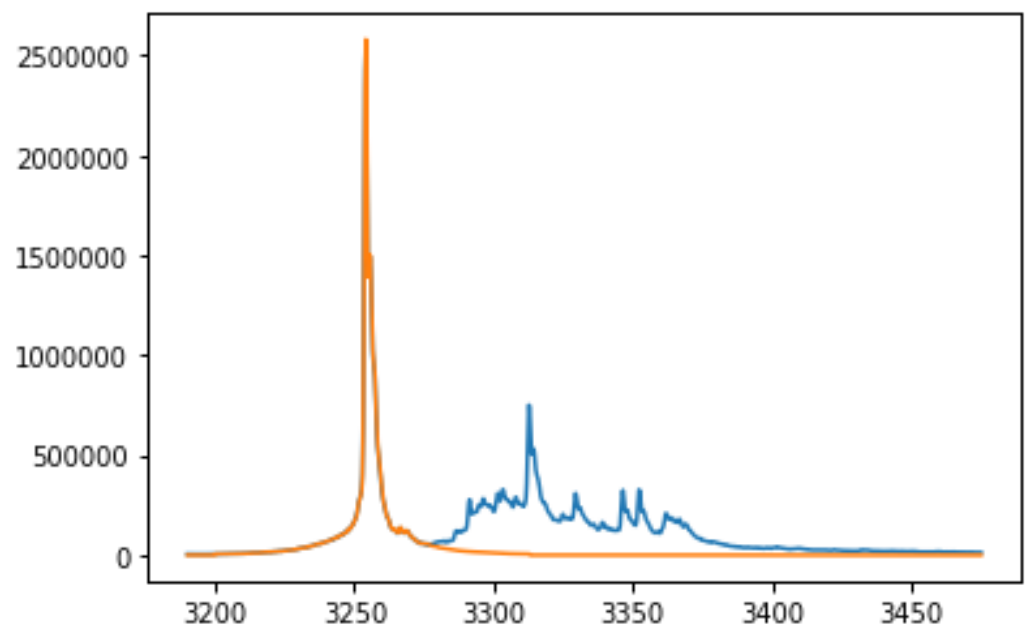




\section{Optimizing peptide intensity values}

In [18]: \#model contains [(mass/charge state),1] for each peptide

model $=$ model $=[[0,1],[36.77156117,1],[37.26341667,1],[40.93858333,1],[42.10$ $05,1],[42.2715,1],[43.77066667,1]$,

$[44.02726233,1],[44.94,1],[46.27244167,1],[49.19433333,1],[51.52511058,1],[5$ $8.6915,1],[59.94783333,1]$,

$[60.94668658,1],[64.70056667,1],[70.125175,1],[70.70325833,1],[71.03535,1],[7$ $4.86914167,1],[75.45411667,1]$,

$[77.95593333,1],[79.54885,1],[81.04521667,1],[84.04284167,1],[85.0397,1],[88.9$ $58625,1],[89.8757,1]$,

$[90.467325,1],[92.29235833,1],[92.46346667,1],[96.2113,1],[98.21211667,1],[10$ $1.141,1],[106.7962167,1],[108.381625,1]$,

$[112.2182417,1],[112.715975,1],[112.9784083,1],[113.220825,1],[115.1363917,1]$, $[120.2250083,1],[122.8079917,1]$,

$[123.8882333,1],[125.1378667,1],[125.399975,1],[129.8079917,1],[132.5582,1],[1$ $33.732225,1],[140.6444833,1]$,

$[144.3133333,1],[146.5642417,1],[159.0785583,1],[165.4203583,1],[166.2484167,1$

], $[209.8499083,1]]$

$\mathrm{mz}$, intensity $=\operatorname{zip}(*$ model $)$

In [26]: \#Shifts the apo feature by the mass of each peptide

def model_spectrum(i):

model_intensity $=\mathrm{np}$.zeros_like $(\mathrm{x} 2)$

for $m, n$ in enumerate(i):

$\mathrm{ms}=\mathrm{mz}[\mathrm{m}]$

mass $=\operatorname{int}(n p \cdot \operatorname{around}(m s * 4))$

model_intensity $+=n p$.absolute $(n) * n p \cdot r o l l(Y M O D E L$, mass)

return model_intensity

\# function to be minimized, gives optimized intensity values

def residual(i):

return np.sqrt(np.sum(np.square(y2-model_spectrum(i))))

In [27]: \#Array containing initial guess for intensity values of each peptide.

\#Starting intensities are generated at random.

randoms $=n p \cdot$ random.dirichlet $(n p \cdot \operatorname{ones}(56)$, size $=1)$

io $=$ randoms.tolist ()

\#minimize the intensity values, starting with the initial guess

res $=\operatorname{minimize}\left(\right.$ residual, $i 0$, method $={ }^{\prime}$ Powell', options $=\{$ 'disp':True $\left.\}\right)$

Optimization terminated successfully.

Current function value: 690439.516746

Iterations: 18

Function evaluations: 13943 
In [21]: \#These are optimized intensity values obtainied from the minimization step abo ve.

optimized_intensities $=[0.99242772,0.02577105,0.06861537,0.04615143$, 0.03080824 ,
0.01544365 ,
0.07427237 ,
0.00957827 ,
0.00553823 ,
0.0245669 ,
0.04826825 ,
0.051237 ,
0.06788056 ,
0.28048348 ,
0.01775716 ,
0.01585566 ,
0.06636562 ,
0.02792444 ,
0.00570916 ,
0.02262009 ,
0.02957831 ,
0.02907774 ,
0.01277072 ,
0.01169131 ,
0.01684148 ,
0.01020779 ,
0.01081695 ,
0.00374415 ,
0.00306709 ,
0.09614033 ,
0.00346409 ,
0.0129223 ,
0.09575206 ,
0.01048002 ,
0.04049952 ,
0.05119921 ,
0.03068795 ,
0.00334174 ,
0.00900667 ,
0.00598633 ,
0.02883885 ,
0.01484173 ,
0.00450266 ,
0.00529158 ,
0.00558654 ,
0.00495927 ,
0.00859375 ,
0.00341611 ,
0.00852671 ,
0.01172867 ,
0.00588204 ,
0.01467977 ,
0.01561106 ,
0.00731439 ,
0.01123291 ,
$0.01452833]$

In [22]: \#Plotting the modeled peptide contributions using the optimized intesnity valu es

mpl.rcParams ['axes.linewidth'] $=1.0$

mpl.rcParams ['xtick.major.width'] $=1.0$

mpl.rcParams ['ytick.major.width'] $=1.0$

fig = plt.figure()

ax1 = fig.add_subplot(111)

ax1.tick_params (direction=' in ' )

results $=[]$

plt.plot $\left(x 2, y 2,{ }^{\prime} k\right.$ ')

for $m, n$ in enumerate(optimized_intensities):

model_intensity $=\mathrm{np} \cdot \operatorname{zeros} \_$like $(\mathrm{x} 2)$

$\mathrm{ms}=\mathrm{mz}[\mathrm{m}]$

mass $=\operatorname{int}(n p \cdot \operatorname{around}(m s * 4))$

model_intensity $+=n p$.absolute $(n) * n p \cdot \operatorname{roll}(Y M O D E L$, mass)

results.append(np.array (model_intensity $[:])$ )

plt.plot (x2, model_intensity)

plt.fill_between( $x 2$, model_intensity, alpha=0.5)

plt.xlim(3225, 3405)

plt.ylim $(0,830000)$

Out [22]: $(0,830000)$

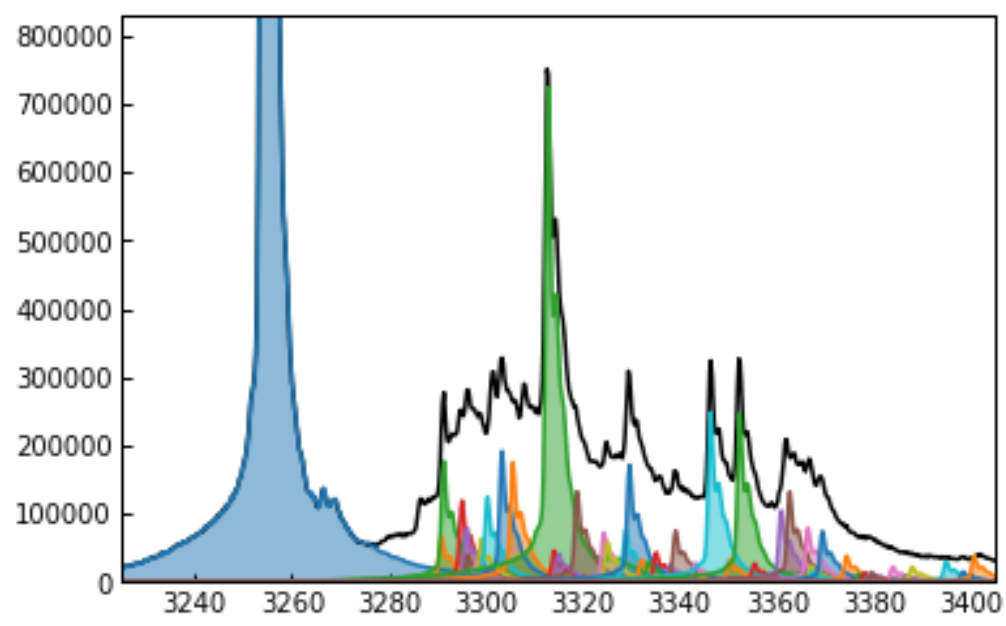

